Homology, Homotopy and Applications, vol.20(1), 2018, pp.117-139

\title{
USING TORSION THEORY TO COMPUTE THE ALGEBRAIC STRUCTURE OF HOCHSCHILD (CO)HOMOLOGY
}

\author{
ESTANISLAO HERSCOVICH
}

(communicated by Claude Cibils)

\begin{abstract}
The aim of this article is to provide explicit formulas for the cup product on the Hochschild cohomology of any nonnegatively graded connected algebra $A$ and for the cap products on the Hochschild homology of $A$ with coefficients in any graded bimodule $M$ at the level of the complexes $\operatorname{Hom}_{A^{\mathrm{e}}}\left(P_{\bullet}, A\right)$ and $M \otimes_{A^{\mathrm{e}}} P_{\bullet}$, resp., where $P_{\bullet}$ is a minimal projective resolution of the $A$-bimodule $A$, based on the $A_{\infty}$-algebra structure of $\mathcal{E} x t_{A}^{\bullet}(k, k)$. We remark that we do not (need to) construct any comparison map between $P_{\bullet}$ and the Hochschild resolution of $A$, or any lift $\Delta: P \rightarrow P \otimes_{A} P$ of the identity of $A$. The main tools we use come from torsion theory of $A_{\infty}$-algebras and of their $A_{\infty}$-bimodules.
\end{abstract}

\section{Introduction}

The goal of this article is to show that, for a nonnegatively graded connected algebra $A$ and a graded $A$-bimodule $M$, it is possible to compute the cup product of the Hochschild cohomology of $A$ and the cap product on the Hochschild homology of $A$ with coefficients in $M$ at the level of the complexes $\operatorname{Hom}_{A^{\mathrm{e}}}\left(P_{\bullet}, A\right)$ and $M \otimes_{A^{\mathrm{e}}} P_{\bullet}$, resp., where $P_{\bullet}$ is a minimal projective resolution of the $A$-bimodule $A$, without constructing any comparison map between $P_{\bullet}$ and the Hochschild resolution of $A$, or any lift $\Delta: P \rightarrow P \otimes_{A} P$ of the identity of $A$, but by making use of the $A_{\infty}$-algebra structure of $\mathcal{E}_{x} t_{A}^{\bullet}(k, k)$. More precisely, the main result of this work is the following (see Theorem 4.3).

Theorem. Let $A$ be a nonnegatively graded connected algebra over a field $k$ and consider $C=\operatorname{Tor}_{\bullet}^{A}(k, k)$ provided with a coaugmented $A_{\infty}$-coalgebra structure whose dual is isomorphic to the augmented $A_{\infty}$-algebra $\mathcal{E}_{x} t_{A}^{\bullet}(k, k)$. Then, there exists a twisted cochain $\tau$ in $\mathcal{H o m}(C, A)$ such that:

This work was also partially supported by UBACYT 20020130200169BA, UBACYT 20020130100533BA, PIP-CONICET 2012-2014 11220110100870, MathAmSud-GR2HOPF, PICT 2011-1510 and PICT 2012-1186.

Received April 7, 2016, revised June 22, 2017, June 26, 2017; published on January 17, 2018.

2010 Mathematics Subject Classification: 16E40, 16E45, 16S37, 16W50, 18 G55.

Key words and phrases: Koszul algebra, Yoneda algebra, homological algebra, dg algebra, $A_{\infty^{-}}$ algebra.

Article available at http://dx.doi.org/10.4310/HHA.2018.v20.n1.a8

Copyright (C) 2018, International Press. Permission to copy for private use granted. 
1. there is a quasi-isomorphism of $A_{\infty}$-algebras from the complex $C^{\bullet}(A, A)$ computing the Hochschild cohomology of $A$ to $\mathcal{H o m}^{\tau}(C, A)$, which, in particular, induces an isomorphism of graded algebras from $H^{\bullet}(A)$ to $H^{\bullet}\left(\mathcal{H o m}^{\tau}(C, A)\right)$;

2. given any graded $A$-bimodule $M$ and using the previous quasi-isomorphism of $A_{\infty}$-algebras, there exists a quasi-isomorphism of $A_{\infty}$-bimodules over $C^{\bullet}(A, A)$ from $M \otimes_{\tau} C$ to the chain complex $C \bullet(A, M)$ computing the Hochschild homology of $A$ with coefficients in $M$, which gives thus an isomorphism of graded bimodules over $H^{\bullet}(A)$ from $H_{\bullet}\left(A \otimes_{\tau} C\right)$ to $H_{\bullet}(A, M)$.

The proof is based on the torsion theory of $A_{\infty}$-algebras and of $A_{\infty}$-bimodules over them by elements satisfying the Maurer-Cartan equation. We also want to remark that there is a very simple condition to check if a given coaugmented $A_{\infty}$-coalgebra structure on $\operatorname{Tor}_{\bullet}^{A}(k, k)$ is dual to the augmented $A_{\infty}$-algebra $\mathcal{E} x t_{A}^{\bullet}(k, k)$ that also provides the twisting cochain $\tau$ (see Theorem 4.2). Moreover, we want to stress that our main result gives explicit expressions of the cup and cap products (see Addendum 4.4). We give, in particular, an application computing the cup and cap products on Hochschild cohomology and homology, resp., of a generalized Koszul algebra $A$ (see Theorem 4.5). The part of this last result concerning Hochschild cohomology was already observed by $\mathrm{Xu}$ and Xiang in [15, Thm. 3.2]. Our proof is, however, completely different. The part concerning Hochschild homology does not seem to have been observed so far. Furthermore, our results can be applied to any nonnegatively graded connected algebras for which the $A_{\infty}$-algebra structure of the Yoneda algebra is known (e.g., the multi-Koszul algebras studied in [8]).

The article is structured as follows. In Section 2 we review the basic definitions and results about the main algebraic objects we shall use: coaugmented $A_{\infty}$-coalgebras and $A_{\infty}$-bimodules over augmented $A_{\infty}$-algebras. For the theory of $A_{\infty}$-algebras we refer the reader to the nice exposition [11], whose convention we shall follow, and to standard references like [10] and [13]. The section is concluded with two constructions that will be used in the sequel: an augmented $A_{\infty}$-algebra $\mathcal{H o m}(C, A)$ from a coaugmented $A_{\infty}$-coalgebra $C$ and an augmented dg algebra $A$, called the convolution $A_{\infty}$-algebra, and, given a $\operatorname{dg} A$-bimodule $M$, an $A_{\infty}$-bimodule $M \otimes C$ over $\mathcal{H o m}(C, A)$.

Section 3 deals with the twisting theory of topological augmented $A_{\infty}$-algebras and of $A_{\infty}$-bimodules over them satisfying a particular discreteness assumption. We surmise that the contents of Subsections 3.2 and 3.4, specially Lemmas 3.4, 3.5, 3.12 and 3.13, might be well-known among the experts, but we could not find any specific reference for them. Our main interest in these constructions is to show that, if $C$ is an Adams connected coaugmented $A_{\infty}$-coalgebra and $\tau$ is a twisting cochain, one can twist the convolution $A_{\infty}$-algebra $\mathcal{H o m}(C, A)$ and the $A_{\infty}$-bimodule $M \otimes C$ over the former. The new constructions are denoted by $\mathcal{H o m}{ }^{\tau}(C, A)$ and $M \otimes_{\tau} C$, respectively. The contents of Subsection 3.3 are standard with the possible exception of Lemma 3.11, which we believe is new even though not completely unexpected. The reason for providing most of the results of this subsection was in order to be able to prove the mentioned lemma, as well as to establish the notation we use in the sequel.

The last Section is divided in three parts. Subsection 4.1 recalls some easy facts about the twisted convolution algebra and the twisted tensor product, and presents a theorem announced by B. Keller at the X ICRA of Toronto, Canada, in 2002 (see 
Theorem 4.2), which is of key importance in the proof of our main result. The latter is proved in Subsection 4.2 (see Theorem 4.3). Finally, Subsection 4.3 provides an application, computing the cup and cap products for the Hochschild (co)homology of a generalized Koszul algebra.

\section{Acknowledgments}

I would like to thank the referee for several comments and suggestions.

\section{Preliminaries on basic algebraic structures}

We recall the following basic facts, which will also establish the notation. From now on, $k$ will denote a field (which we also consider as a unitary graded ring concentrated in degree zero). We will denote by $\mathbb{N}$ the set of (strictly) positive integers and by $\mathbb{N}_{0}=\{0\} \cup \mathbb{N}$. In this article $A_{\infty}$-algebras (and coalgebras) and their (co)modules will be graded with respect to $G=\mathbb{Z} \times \mathbb{Z}$, where the first grading is called (co)homological and the second one is called Adams. When applying the Koszul sign rule, we will only take the cohomological degree into account (for a nice exposition on basic homological algebra of $\mathrm{dg}$ modules over dg algebras and a detailed account on the sign rule we refer to [1]). We shall denote the cohomological degree of a homogeneous element $x$ by $\operatorname{deg} x$. We recall that a family of linear maps $\left\{f_{i}: M \rightarrow M_{i}\right\}_{i \in \mathbb{N}}$, where $M$ and $M_{i}$, for $i \in \mathbb{N}$, are vector spaces, is called locally finite if, for all $m \in M$, there exists a finite subset $S \subseteq \mathbb{N}$, which depends on $m$, such that $f_{i}(m)$ vanishes for all $i \in \mathbb{N} \backslash S$. Given $n_{0} \in \mathbb{Z}$ and $V=\oplus_{(n, m) \in \mathbb{Z}^{2}} V^{(n, m)}$ any graded object (decorated perhaps with further adjectives), we shall denote by $V\left[n_{0}\right]$ the shift of $V$, defined as $V\left[n_{0}\right]^{(n, m)}=V^{\left(n+n_{0}, m\right)}$ for all $n, m \in \mathbb{Z}$, and by $s_{V, n_{0}}: V \rightarrow V\left[n_{0}\right]$ the morphism of cohomological degree $-n_{0}$, called the suspension on $V$ of degree $n_{0}$, whose underlying set-theoretic map is the identity. In this work we shall never consider shifts of the Adams degree. If $n_{0}=1$ we will just write $s_{V}$ and call it the suspension on $V$. We shall say that a graded vector space $V=\oplus_{(m, n) \in \mathbb{Z}^{2}} V^{(m, n)}$ over $k$ is Adams connected if $V^{(0,0)}=k$ and $V^{\wedge}=\oplus_{(m, n) \in \mathbb{Z}^{2} \backslash\{(0,0)\}} V^{(m, n)}$ is concentrated in either strictly positive or strictly negative Adams degrees, and each homogeneous component of $V^{\wedge}$ of a fixed Adams degree $d$ (but including all cohomological degrees) is locally finite dimensional ( $c f$. [12, Def. 2.1]). By changing if necessary the Adams grading of $V$ by its opposite, we may (and shall) assume without loss of generality that an Adams connected graded vector space $V$ satisfies that $V^{\wedge}$ is concentrated in Adams positive degrees.

\subsection{Generalities on $A_{\infty}$-(co)algebras}

For the general theory of $A_{\infty}$-algebras and $A_{\infty}$-coalgebras we refer to [10, Chs. 1$3]$, or [13, Ch. 3], even though we shall not follow the sign conventions of those works. The precise definition and sign conventions that we shall use of augmented $A_{\infty}$-algebras, morphisms among them, their bar construction and modules are given in the very nice exposition [11], to which we refer. We will present, however, the basic definitions for the reader's convenience.

Let $A$ be a graded vector space provided with two maps $\eta_{A}: k \rightarrow A$ and $\epsilon_{A}: A \rightarrow k$ of degree zero, that we call the unit and the augmentation of $A$, respectively. Set $I_{A}=\operatorname{Ker}\left(\epsilon_{A}\right)$ and $1_{A}=\eta\left(1_{k}\right)$. This defines an isomorphism of graded vector spaces 
$A \simeq k \cdot 1_{A} \oplus I_{A}$. An augmented $A_{\infty}$-algebra is a coderivation $B_{A}$ of cohomological degree 1 on the coaugmented graded coalgebra $T\left(I_{A}[1]\right)=\oplus_{i \in \mathbb{N}_{0}} I_{A}[1]^{\otimes i}$ with the coproduct given by deconcatenation, the obvious counit $\epsilon_{T}$ and coaugmentation $\eta_{T}$, such that $B_{A} \circ \eta_{T}=0$ and $B_{A} \circ B_{A}=0$. This coaugmented dg coalgebra is called the bar construction of $A$ and is denoted by $B^{+}(A)$.

Taking into account that $B^{+}(A)$ is a coaugmented tensor graded coalgebra, it is cocomplete and cofree, so the coderivation $B_{A}$ is uniquely determined by $\pi_{1} \circ B_{A}$, where $\pi_{1}: B^{+}(A) \rightarrow I_{A}[1]$ is the canonical projection (see [10, Lemme 1.1.2.2]). Let us write this composition map by $b=\sum_{i \in \mathbb{N}} b_{i}$, where $b_{i}: I_{A}[1]^{\otimes i} \rightarrow I_{A}[1]$. For $i \in$ $\mathbb{N}$, define $\bar{m}_{i}: I_{A}^{\otimes i} \rightarrow I_{A}$ by means of $b_{i}=-s_{I_{A}} \circ m_{i} \circ\left(s_{I_{A}}^{\otimes i}\right)^{-1}$, and extend it to a map $m_{i}: A^{\otimes i} \rightarrow A$ given by the composition of $\bar{m}_{i} \circ p^{\otimes i}$ and the canonical inclusion $I_{A} \rightarrow A$ if $i \neq 2$, where $p: A \rightarrow I_{A}$ is the canonical projection, and by $\left.m_{2}\right|_{I_{A}^{\otimes 2}}=\bar{m}_{2}$ and $m_{2}\left(1_{A} \otimes a\right)=a=m_{2}\left(a \otimes 1_{1}\right)$ for all $a \in A$. If the reader translates the previous definition of augmented $A_{\infty}$-algebra into a condition about the maps $\left\{m_{i}\right\}_{i \in \mathbb{N}}$, he will find precisely the classical notion appearing in [11]. ${ }^{1}$

A morphism of augmented $A_{\infty}$-algebras $f_{\bullet}: A \rightarrow B$ between two augmented $A_{\infty^{-}}$ algebras $A$ and $B$ is a morphism of coaugmented dg coalgebras $B^{+}(f): B^{+}(A) \rightarrow$ $B^{+}(B)$. Since $B^{+}(B)$ is a coaugmented tensor graded coalgebra, the morphism $B^{+}(f)$ is uniquely determined by its composition with the canonical projection $B^{+}(B) \rightarrow$ $I_{B}[1]$, that we write $F=\sum_{i \in \mathbb{N}} F_{i}$, where $F_{i}: I_{A}[1]^{\otimes i} \rightarrow I_{B}$ [1]. Set $\bar{f}_{i}: I_{A}^{\otimes i} \rightarrow I_{B}$ by means of $F_{i}=s_{I_{B}} \circ \bar{f}_{i} \circ\left(s_{I_{A}}^{\otimes i}\right)^{-1}$, for $i \in \mathbb{N}$, and define $f_{i}: A^{\otimes i} \rightarrow B$ as the composition of $\bar{f}_{i} \circ p^{\otimes i}$ and the canonical inclusion $I_{B} \rightarrow B$ if $i \neq 1$, where $p: A \rightarrow I_{A}$ is the canonical projection, and by $f_{1}\left(1_{A}\right)=1_{B}$ and $\left.f_{1}\right|_{I_{A}}=\bar{f}_{1}$. The translation of the definition of morphism of augmented $A_{\infty}$-algebras into a condition about the maps $\left\{f_{i}\right\}_{i \in \mathbb{N}}$ gives precisely the classical notion that appears in [11].

We shall briefly recall the dual notions, because they are no so common in the literature. Let $C$ be a graded vector space provided two maps $\epsilon_{C}: C \rightarrow k$ and $\eta_{C}: k \rightarrow$ $C$ of degree zero, called the counit and the coaugmentation of $C$, respectively. Define $1_{C}=\eta_{C}\left(1_{k}\right)$ and $J_{C}=\operatorname{Coker}\left(\eta_{C}\right)$, and consider the isomorphism of graded vector spaces $C \simeq k .1_{C} \oplus J_{C}$ via the identification $J_{C} \simeq \operatorname{Ker}\left(\epsilon_{C}\right)$.

A coaugmented $A_{\infty}$-coalgebra is a derivation $D_{C}$ of cohomological degree 1 on the augmented graded tensor algebra $T\left(J_{C}[-1]\right)$ provided with the concatenation, and the obvious unit $\eta_{T}$ and augmentation $\epsilon_{T}$, such that $\epsilon_{T} \circ D_{C}=0$ and $D_{C} \circ D_{C}=0$. The previous augmented dg algebra is called the cobar construction of $C$ and is typically denoted by $\Omega^{+}(C)$.

Since $\Omega^{+}(C)$ is a free graded algebra, $D_{C}$ is uniquely determined by its restriction to $J_{C}[-1]$, which we denote by $d=\sum_{i \in \mathbb{N}} d_{i}$ for $d_{i}: J_{C}[-1] \rightarrow J_{C}[-1]^{\otimes i}$. Set $\bar{\Delta}_{i}: J_{C} \rightarrow J_{C}^{\otimes i}$ by means of $d_{i}=(-1)^{i}\left(s_{J_{C}[-1]}^{\otimes i}\right)^{-1} \circ \bar{\Delta}_{i} \circ s_{J_{C}[-1]}$, and define $\Delta_{i}: C \rightarrow$ $C^{\otimes i}$ as the composition of the canonical projection $C \rightarrow J_{C}, \bar{\Delta}_{i}$ and the inclusion $J_{C}^{\otimes i} \rightarrow C^{\otimes i}$ if $i \neq 2$, and as $\Delta_{2}\left(1_{C}\right)=1_{C} \otimes 1_{C}$ and $\Delta_{2}(\bar{c})=\bar{\Delta}_{2}(\bar{c})+1_{C} \otimes \bar{c}+\bar{c} \otimes 1_{C}$ for $\bar{c} \in J_{C}$. Then the collection of maps $\Delta_{i}: C \rightarrow C^{\otimes i}$ for $i \in \mathbb{N}$ is locally finite, each

\footnotetext{
${ }^{1}$ Unfortunately, the choice of sign appearing in the bar construction of Section 9 of [11] is mistaken, for it is not compatible with their Definition 2.1 if they want their Lemma 9.1 to hold. Using the notation of that article, a coherent choice of sign is given by taking $\bar{m}_{n}=-m_{n}$, and by replacing $\left(\left|a_{j+t}\right|+1\right)$ by $\left|a_{j+t}\right|$ in their definition of $w$ at p. 22 .
} 
of homological degree $i-2$ and Adams degree zero, satisfying the following identities

$$
\sum_{(r, s, t) \in \mathcal{I}_{n}}(-1)^{r s+t}\left(\mathrm{id}_{C}^{\otimes r} \otimes \Delta_{s} \otimes \mathrm{id}_{C}^{\otimes t}\right) \circ \Delta_{r+1+t}=0,
$$

for $n \in \mathbb{N}$, where $\mathcal{I}_{n}=\left\{(r, s, t) \in \mathbb{N}_{0} \times \mathbb{N} \times \mathbb{N}_{0}: r+s+t=n\right\},\left(\operatorname{id}_{C}^{\otimes r} \otimes \epsilon_{C} \otimes \operatorname{id}_{C}^{\otimes t}\right) \circ$ $\Delta_{i}$ vanishes for all $i \neq 2$ and $r, t \geqslant 0$ such that $r+1+t=i,\left(\operatorname{id}_{C} \otimes \epsilon_{C}\right) \circ \Delta_{2}=\operatorname{id}_{C}=$ $\left(\epsilon_{C} \otimes \mathrm{id}_{C}\right) \circ \Delta_{2}, \epsilon_{C} \circ \eta_{C}=\mathrm{id}_{k}, \Delta_{2} \circ \eta_{C}\left(1_{k}\right)=\eta_{C}\left(1_{k}\right)^{\otimes 2}$, and $\Delta_{i} \circ \eta_{C}\left(1_{k}\right)=0$ for all $i \in \mathbb{N} \backslash\{2\}$. Reciprocally, starting from a locally finite collection of maps $\Delta_{i}: C \rightarrow$ $C^{\otimes i}$ fulfilling the previous properties we obtain a coaugmented $A_{\infty}$-coalgebra structure. A coaugmented $A_{\infty}$-coalgebra $C$ is called minimal if $\Delta_{1}=0$.

Note that a coaugmented $A_{\infty}$-coalgebra $C$ is also a complex of vector spaces for the differential $\Delta_{1}$. Moreover, a coaugmented dg coalgebra structure on $C$ is equivalent to a coaugmented $A_{\infty}$-coalgebra structure with vanishing higher comultiplications $\Delta_{n}$ for $n \geqslant 3$, where the differential is $\Delta_{1}$ and the coproduct is $\Delta_{2}$. Note that our definition of the cobar construction for the special case of a coaugmented dg coalgebra coincides with the one given in [11, Section 8].

Given two coaugmented $A_{\infty}$-coalgebras $C$ and $C^{\prime}$, a morphism $f_{\bullet}: C \rightarrow C^{\prime}$ is a morphism of augmented dg algebras $\Omega^{+}\left(f_{\bullet}\right): \Omega^{+}(C) \rightarrow \Omega^{+}\left(C^{\prime}\right)$ of the cobar constructions. Since $\Omega^{+}(C)$ is a free graded algebra, such a morphism is completely determined by its restriction to $J_{C}[-1]$, which we denote by $F=\sum_{i \in \mathbb{N}} F_{i}$, where $F_{i}: J_{C}[-1] \rightarrow$ $J_{C^{\prime}}[-1]^{\otimes i}$. Define $\bar{f}_{i}: J_{C} \rightarrow J_{C^{\prime}}^{\otimes i}$ by $F_{i}=(-1)^{i+1}\left(s_{J_{C^{\prime}}[-1]}^{\otimes i}\right)^{-1} \circ \bar{f}_{i} \circ s_{J_{C}[-1]}$, for $i \in \mathbb{N}$, and set $f_{i}: C \rightarrow\left(C^{\prime}\right)^{\otimes i}$ as the composition of the canonical projection $C \rightarrow J_{C}, \bar{f}_{i}$ and the inclusion $J_{C^{\prime}}^{\otimes i} \rightarrow\left(C^{\prime}\right)^{\otimes i}$ if $i \neq 1$, and $f_{1}\left(1_{C}\right)=1_{C}$ and $f_{1}(\bar{c})=\bar{f}_{1}(\bar{c})$ for $\bar{c} \in J_{C}$. Then $f_{i}: C \rightarrow\left(C^{\prime}\right)^{\otimes i}$ is a locally finite collection of maps, each of homological degree $i-1$ and Adams degree zero for $i \in \mathbb{N}$, such that

$$
\sum_{(r, s, t) \in \mathcal{I}_{n}}(-1)^{r s+t}\left(\operatorname{id}_{D}^{\otimes r} \otimes \Delta_{s}^{D} \otimes \mathrm{id}_{D}^{\otimes t}\right) \circ f_{r+1+t}=\sum_{q \in \mathbb{N}} \sum_{i \in \mathbb{N}^{q}, n}(-1)^{w^{\prime}}\left(f_{i_{1}} \otimes \cdots \otimes f_{i_{q}}\right) \circ \Delta_{q}^{C},
$$

where $w^{\prime}=\sum_{j=1}^{q}(j-1)\left(i_{j}+1\right)$ and $\mathbb{N}^{q, n}$ is the subset of elements $\bar{i}$ of $\mathbb{N}^{q}$ satisfying that $|\bar{i}|=i_{1}+\cdots+i_{q}=n, \epsilon_{C^{\prime}} \circ f_{1}=\epsilon_{C},\left(\operatorname{id}_{C^{\prime}}^{\otimes(j-1)} \otimes \epsilon_{C^{\prime}} \otimes \operatorname{id}_{C^{\prime}}^{\otimes(i-j)}\right) \circ f_{i}$ vanishes for all $i \geqslant 2$ and $j \in\{1, \ldots, i\}, f_{1} \circ \eta_{C}=\eta_{C^{\prime}}$ and $f_{i} \circ \eta_{C}$ vanishes for $i \geqslant 2$. Reciprocally, starting from a locally finite collection of maps $f_{i}: C \rightarrow\left(C^{\prime}\right)^{\otimes i}$ fulfilling the previous properties we obtain a morphism of coaugmented $A_{\infty}$-coalgebras.

Notice that $f_{1}$ is also a morphism of dg vector spaces for the underlying structures on $C$ and $C^{\prime}$. A morphism of coaugmented $A_{\infty}$-coalgebras is strict if $f_{i}$ vanishes for $i \geqslant 2$ and it is called a quasi-isomorphism if the map $f_{1}$ is so. We remark that our definition of $\Omega^{+}\left(f_{\bullet}\right)$ agrees with the corresponding one given in [11, Section 8], for coaugmented dg coalgebras in the case the morphism $f_{\bullet}$ is further assumed to be strict.

If $f_{\bullet}: C \rightarrow C^{\prime}$ and $g_{\bullet}: C^{\prime} \rightarrow D$ are morphisms of coaugmented $A_{\infty}$-coalgebras, we can consider their composition $\Omega^{+}\left(g_{\bullet}\right) \circ \Omega^{+}\left(f_{\bullet}\right)$. Using the comments in the previous paragraphs we see that $\Omega^{+}\left(g_{\bullet}\right) \circ \Omega^{+}\left(f_{\bullet}\right)=\Omega^{+}\left(h_{\bullet}\right)$ where $\left\{h_{n}: C \rightarrow D^{\otimes n}\right\}_{n \in \mathbb{N}}$ is of the form

$$
h_{n}=\sum_{q \in \mathbb{N}} \sum_{\bar{i} \in \mathbb{N}^{q}, n}(-1)^{w^{\prime}}\left(g_{i_{1}} \otimes \cdots \otimes g_{i_{q}}\right) \circ f_{q},
$$

and $w^{\prime}=\sum_{j=1}^{q}(j-1)\left(i_{j}+1\right)$. 


\subsection{Generalities on $A_{\infty}$-bimodules}

For the following definitions we refer to [10, Ch. 2, Section 5], even though our sign conventions are distinct. Given an augmented $A_{\infty}$-algebra $A$, an $A_{\infty}$-bimodule over $A$ is a graded vector space $M$ and a bicoderivation $B_{M}$ on the graded counitary bicomodule $B^{+}(A) \otimes M[1] \otimes B^{+}(A)$ over $B^{+}(A)$ such that $B_{M} \circ B_{M}=0$. We shall denote the previous bicomodule by $B^{+}(A, M, A)$. Since $B^{+}(A) \otimes M[1] \otimes B^{+}(A)$ is a cofree graded bicomodule, a bicoderivation is uniquely determined by its composition with $\epsilon_{B^{+}(A)} \otimes \operatorname{id}_{M[1]} \otimes \epsilon_{B^{+}(A)}$, which is a sum of mappings of the form $b_{p, q}: I_{A}[1]^{\otimes p} \otimes$ $M[1] \otimes I_{A}[1]^{\otimes p} \rightarrow M[1]$, for $p, q \in \mathbb{N}_{0}$. Define $m_{p, q}^{M}: A^{\otimes p} \otimes M \otimes A^{\otimes q} \rightarrow M$ as the composition of the canonical projection $A^{\otimes p} \otimes M \otimes A^{\otimes q} \rightarrow I_{A}^{\otimes p} \otimes M \otimes I_{A}^{\otimes q}$ and $-s_{M}^{-1} \circ b_{p, q} \circ\left(s_{I_{A}}^{\otimes p} \otimes s_{M} \otimes s_{I_{A}}^{\otimes q}\right)$ if $p+q \neq 1$, and by $m_{0,1} \circ\left(\operatorname{id}_{M} \otimes \eta_{A}\right)=\mathrm{id}_{M}=m_{1,0} \circ$ $\left(\eta_{A} \otimes \mathrm{id}_{M}\right)$ as well as the equalities $m_{0,1} \circ\left(\operatorname{id}_{M} \otimes i_{A}\right)=-s_{M}^{-1} \circ b_{0,1} \circ\left(s_{M} \otimes s_{I_{A}}\right)$ and $m_{1,0} \circ\left(i_{A} \otimes \operatorname{id}_{M}\right)=-s_{M}^{-1} \circ b_{1,0} \circ\left(s_{I_{A}} \otimes s_{M}\right)$, where $i_{A}: I_{A} \rightarrow A$ is the canonical inclusion.

They satisfy the identity

$$
\sum_{(r, s, t) \in \mathcal{I}_{n^{\prime}+n^{\prime \prime}+1}}(-1)^{r+s t} \tilde{m}_{r, t}^{M} \circ\left(\mathrm{id}^{\otimes r} \otimes \tilde{m}_{s} \otimes \mathrm{id}^{\otimes t}\right)=0,
$$

for all $n^{\prime}, n^{\prime \prime} \in \mathbb{N}_{0}$, where we recall that $\mathcal{I}_{n}=\left\{(r, s, t) \in \mathbb{N}_{0} \times \mathbb{N} \times \mathbb{N}_{0}: r+s+t=n\right\}$, and where $\tilde{m}_{s}$ is interpreted as the corresponding multiplication map $m_{s}$ of $A$ if either $r+s \leqslant n^{\prime}$ or $s+t \leqslant n^{\prime \prime}$, and it is understood as $m_{n^{\prime}-r, n^{\prime \prime}-t}^{M}$ else. In the first case, $\tilde{m}_{r, t}^{M}$ is $m_{n^{\prime}-s+1, n^{\prime \prime}}^{M}$ if $r+s \leqslant n^{\prime}$ or $m_{n^{\prime}, n^{\prime \prime}-s+1}^{M}$ if $s+t \leqslant n^{\prime \prime}$, and it is $m_{r, t}^{M}$ else. We also have that $m_{p, q}^{M} \circ\left(\mathrm{id}^{\otimes r} \otimes \eta_{A} \otimes \mathrm{id}^{\otimes t}\right)$ vanishes for $r \neq p$ and $(p, q) \notin\{(0,1),(1,0)\}$, and that $m_{1,0}^{M} \circ\left(\eta_{A} \otimes \operatorname{id}_{M}\right)=\operatorname{id}_{M}=m_{0,1}^{M} \circ\left(\operatorname{id}_{M} \otimes \eta_{A}\right)$. Reciprocally, given any collection of maps $m_{p, q}^{M}: A^{\otimes p} \otimes M \otimes A^{\otimes q} \rightarrow M$ fulfilling the previous properties, it defines an $A_{\infty}$-bimodule structure on $M$ over $A$. Note that an augmented $A_{\infty}$-algebra is also an $A_{\infty}$-bimodule for the structure maps $m_{p, q}=m_{p+q+1}$, where $p, q \in \mathbb{N}_{0}$.

If $M$ and $N$ are two $A_{\infty}$-bimodules, a morphism $f_{\bullet}, \bullet$ of $A_{\infty}$-bimodules from $M$ to $N$ is a morphism of counitary dg bicomodules $B^{+}\left(f_{\bullet, \bullet}\right): B^{+}(A, M, A) \rightarrow B^{+}(A, N, A)$. Since $B^{+}(A) \otimes M[1] \otimes B^{+}(A)$ is a cofree graded bicomodule, $B^{+}\left(f_{\bullet \bullet \bullet}\right)$ is uniquely determined by its composition with $\epsilon_{B^{+}(A)} \otimes \mathrm{id}_{N[1]} \otimes \epsilon_{B^{+}(A)}$, which is a sum of maps $F_{p, q}: I_{A}^{\otimes p} \otimes M[1] \otimes I_{A}[1]^{\otimes q} \rightarrow N[1]$. Define $f_{p, q}^{M}: A^{\otimes p} \otimes M \otimes A^{\otimes q} \rightarrow N$ as the composition of the canonical projection $A^{\otimes p} \otimes M \otimes A^{\otimes q} \rightarrow I_{A}^{\otimes p} \otimes M \otimes I_{A}^{\otimes q}$ and $s_{N}^{-1}$ 。 $f_{p, q} \circ\left(s_{I_{A}}^{\otimes p} \otimes s_{M} \otimes s_{I_{A}}^{\otimes q}\right)$. The collection of morphisms $f_{p, q}: A^{\otimes p} \otimes M \otimes A^{\otimes q} \rightarrow N$ satisfy

$$
\begin{aligned}
& \sum_{(r, s, t) \in \mathcal{I}_{n^{\prime}+n^{\prime \prime}+1}}(-1)^{r+s t} f_{r^{\prime}, t^{\prime}} \circ\left(\mathrm{id}^{\otimes r} \otimes \tilde{m}_{s} \otimes \mathrm{id}^{\otimes t}\right) \\
& \quad=\sum_{(a, k, l, b) \in \mathbb{N}_{0, n^{\prime}, n^{\prime \prime}}}(-1)^{b(-k-l)} m_{a, b}^{N} \circ\left(\operatorname{id}_{A}^{\otimes a} \otimes f_{k, l} \otimes \mathrm{id}_{A}^{\otimes b}\right),
\end{aligned}
$$

for all $n^{\prime}, n^{\prime \prime} \in \mathbb{N}_{0}$, where $\mathbb{N}_{0, n^{\prime}, n^{\prime \prime}}$ is the subset of $\mathbb{N}_{0}^{4}$ of elements $(a, k, l, b)$ such that $a+k=n^{\prime}$ and $l+b=n^{\prime \prime}$, and where we should understand $\tilde{m}_{s}$ as $m_{s}^{A}$ if either $r+s \leqslant$ $n^{\prime}$ or $s+t \leqslant n^{\prime \prime}$, or as $m_{n^{\prime}-r, n^{\prime \prime}-t}^{M}$ else. The indices $\left(r^{\prime}, t^{\prime}\right)$ are completely determined from the previous cases. We also have that $f_{p, q} \circ\left(\mathrm{id}^{\otimes r} \otimes \eta_{A} \otimes \mathrm{id}^{\otimes t}\right)$ vanishes for $r \neq p$ 
and $(p, q) \notin\{(0,0)\}$. Reciprocally, given any collection of maps $f_{p, q}^{M}: A^{\otimes p} \otimes M \otimes$ $A^{\otimes q} \rightarrow N$ fulfilling the previous properties, it defines a morphism of $A_{\infty}$-bimodules from $M$ to $N$ over $A$. We say that it is strict if $f_{p, q}$ vanishes for all $(p, q) \neq(0,0)$.

The composition of two morphisms $f_{\bullet, \bullet}: M \rightarrow N$ and $g_{\bullet \bullet \bullet}: N \rightarrow P$ is defined by the usual formula $B^{+}\left(g_{\bullet}, \bullet\right) \circ B^{+}\left(f_{\bullet}, \bullet\right)$. It is easy to see that the composition is given by $B^{+}\left(h_{\bullet, \bullet}\right)$, where

$$
h_{p, q}=\sum_{(a, k, l, b) \in \mathbb{N}_{0, p, q}}(-1)^{b(-k-l)} g_{a, b} \circ\left(\mathrm{id}_{A}^{\otimes a} \otimes f_{k, l} \otimes \mathrm{id}_{A}^{\otimes b}\right) .
$$

If $f_{\bullet}: A^{\prime} \rightarrow A$ is a morphism of augmented $A_{\infty}$-algebras and $N$ is an $A_{\infty}$-bimodule over $A$ whose bar construction $B^{+}(A, N, A)$ has coderivation $B_{N}$, then $N$ can be regarded as an $A_{\infty}$-bimodule over $A^{\prime}$ with the unique coderivation $B_{N}^{\prime}$ determined by $\left(\epsilon_{B^{+}(A)} \otimes \operatorname{id}_{N[1]} \otimes \epsilon_{B^{+}(A)}\right) \circ B_{N} \circ\left(B^{+}\left(f_{\bullet}\right) \otimes \operatorname{id}_{N[1]} \otimes B^{+}\left(f_{\bullet}\right)\right)$. Note that

$$
B_{N} \circ\left(B^{+}\left(f_{\bullet}\right) \otimes \operatorname{id}_{N[1]} \otimes B^{+}\left(f_{\bullet}\right)\right)=\left(B^{+}\left(f_{\bullet}\right) \otimes \operatorname{id}_{N[1]} \otimes B^{+}\left(f_{\bullet}\right)\right) \circ B_{N}^{\prime},
$$

for the compositions of both members with $\left(\epsilon_{B^{+}(A)} \otimes \mathrm{id}_{N[1]} \otimes \epsilon_{B^{+}(A)}\right)$ coincide. Equivalently, if $m_{\bullet, \bullet}$ are the multiplications of $N$ over $A$, then the structure of an $A_{\infty}$-bimodule over $A^{\prime}$ is given by

$$
m_{p, q}^{\prime}=\sum_{r, s \in \mathbb{N}_{0}} \sum_{(\bar{i}, \bar{j}) \in \mathbb{N}^{r, p} \times \mathbb{N}^{s, q}}(-1)^{\varepsilon} m_{r, s} \circ\left(f_{i_{1}} \otimes \cdots \otimes f_{i_{r}} \otimes \mathrm{id}_{N} \otimes f_{j_{1}} \otimes \cdots \otimes f_{j_{s}}\right),
$$

where we recall that $\mathbb{N}^{m, n}$ is the subset of $\mathbb{N}^{m}$ of elements $\bar{i}=\left(i_{1}, \ldots, i_{m}\right)$ such that $|\bar{i}|=i_{1}+\cdots+i_{m}=n$, and $\varepsilon=\sum_{u=1}^{r}(r+s+1-u)\left(i_{u}-1\right)+\sum_{u=1}^{s}(s-u)\left(j_{u}-1\right)$. We shall denote the $A_{\infty}$-bimodule over $A^{\prime}$ with structure maps $m_{\bullet}^{\prime}$. by $f_{\bullet} N$.

\subsection{Two constructions}

If $C$ is a coaugmented $A_{\infty}$-coalgebra and $A$ is an augmented dg algebra, the $\mathrm{dg}$ vector space $\mathcal{H}=\mathcal{H o m}(C, A)$ has the structure of an augmented $A_{\infty}$-algebra, which we call the convolution $A_{\infty}$-algebra, where $m_{1}^{\mathcal{H}}$ is given by the usual differential $d_{A}$ 。 $\phi-(-1)^{\operatorname{deg} \phi} \phi \circ \Delta_{1}^{C}$,

$$
m_{n}^{\mathcal{H}}\left(\phi_{1} \otimes \cdots \otimes \phi_{n}\right)=(-1)^{n\left(\operatorname{deg} \phi_{1}+\cdots+\operatorname{deg} \phi_{n}+1\right)} \mu_{A}^{(n)} \circ\left(\phi_{1} \otimes \cdots \otimes \phi_{n}\right) \circ \Delta_{n}^{C},
$$

for $n \geqslant 2,1_{\mathcal{H o m}(C, A)}=\eta_{A} \circ \epsilon_{C}$ and $\epsilon_{\mathcal{H} o m(C, A)}(\phi)=\epsilon_{A} \circ \phi \circ \eta_{C}\left(1_{k}\right)$.

If $f_{\bullet}: C \rightarrow D$ is a morphism of coaugmented $A_{\infty}$-coalgebras, then the collection of morphisms

$$
\left(f_{n}\right)_{*}: \mathcal{H o m}(D, A)^{\otimes n} \rightarrow \mathcal{H o m}(C, A),
$$

for $n \in \mathbb{N}$, of graded vector spaces of cohomological degree $1-n$ given by $\left(f_{1}\right)_{*}(\phi)=$ $\phi \circ f_{1}$, and by

$$
\left(f_{n}\right)_{*}\left(\phi_{1} \otimes \cdots \otimes \phi_{n}\right)=(-1)^{(n-1)\left(\operatorname{deg} \phi_{1}+\cdots+\phi_{n}\right)} \mu_{A}^{(n)} \circ\left(\phi_{1} \otimes \cdots \otimes \phi_{n}\right) \circ f_{n},
$$

for $n \geqslant 2$, is a morphism of augmented $A_{\infty}$-algebras. We remark that the previous construction defines an augmented $A_{\infty}$-algebra structure on the graded dual $C^{\#}$ of $C$.

If $M$ is a $\operatorname{dg} A$-bimodule over an augmented dg algebra $A$ and $C$ is a coaugmented 
$A_{\infty}$-coalgebra, then $M \otimes C$ is an $A_{\infty}$-bimodule over $\mathcal{H o m}(C, A)$ with the structure morphisms given by $m_{0,0}^{M \otimes C}=d_{M} \otimes \operatorname{id}_{C}+\operatorname{id}_{M} \otimes \Delta_{1}^{C}$, and, for $p+q \geqslant 1$,

$$
\begin{aligned}
m_{p, q}^{M \otimes C} & \left(\phi_{1} \otimes \cdots \otimes \phi_{p} \otimes(m \otimes c) \otimes \psi_{1} \otimes \cdots \otimes \psi_{q}\right) \\
& =(-1)^{\epsilon^{\prime}}\left(\phi_{1}\left(c_{(q+2)}\right) \cdots \phi_{p}\left(c_{(q+p+1)}\right)\right) \cdot m \cdot\left(\psi_{1}\left(c_{(1)}\right) \cdots \psi_{q}\left(c_{(q)}\right)\right) \otimes c_{(q+1)},
\end{aligned}
$$

where $\Delta_{p+q+1}^{C}(c)=c_{(1)} \otimes \cdots \otimes c_{(p+q+1)}$, and

$$
\begin{aligned}
& \epsilon^{\prime}=p q+\operatorname{deg} c \operatorname{deg} m+(p+q+1) \sum_{i=1}^{p} \operatorname{deg} \phi_{i}+\sum_{\substack{1 \leqslant i \leqslant p \\
1 \leqslant 2 \leqslant i^{\prime} \leqslant q+i}} \operatorname{deg} c_{\left(i^{\prime}\right)} \operatorname{deg} \phi_{i} \\
& +\sum_{1 \leqslant j \leqslant q} \operatorname{deg} c_{\left(j^{\prime}\right)} \operatorname{deg} \psi_{j}+\left(\operatorname{deg} m+\sum_{i=1}^{p} \operatorname{deg} c_{(q+1+i)}+\sum_{j=1}^{q} \operatorname{deg} \psi_{j}\right)\left(\sum_{j=1}^{q+1} \operatorname{deg} c_{(j)}\right) . \\
& 1 \leqslant j^{\prime}<j
\end{aligned}
$$

It is rather long but straightforward to prove that it is indeed an $A_{\infty}$-bimodule over $\mathcal{H o m}(C, A)$. If $M$ is only a left (resp., right) dg module over $A$, we can (and shall) regard it as a $\operatorname{dg} A$-bimodule by means of the augmentation $\epsilon_{A}$, i.e. a.m. $a^{\prime}=$ $\epsilon_{A}\left(a^{\prime}\right) a . m$ (resp., a.m. $\left.a^{\prime}=\epsilon_{A}(a) m . a^{\prime}\right)$, so we may apply the previous construction. It will be denoted by $M_{\epsilon_{A}}$ (resp., $\left.\epsilon_{A} M\right)$.

If $g: M \rightarrow N$ is a morphism of $\operatorname{dg} A$-bimodules over an augmented $\operatorname{dg}$ algebra $A$ and $C$ is a coaugmented $A_{\infty}$-coalgebra, then the map $g \otimes \mathrm{id}_{C}: M \otimes C \rightarrow N \otimes C$ is a strict morphism of $A_{\infty}$-bimodules over $\mathcal{H o m}(C, A)$.

Let $f_{\bullet}: C \rightarrow D$ be a morphism of coaugmented $A_{\infty}$-coalgebras and let $A$ be an augmented $\mathrm{dg}$ algebra. It induces a morphism of augmented $A_{\infty}$-algebras $\left(f_{\bullet}\right)_{*}: \mathcal{H o m}(D, A) \rightarrow \mathcal{H o m}(C, A)$, as seen in (4). In particular, given any $\operatorname{dg} A$-bimodule $M$, this allows to consider $M \otimes C$ as an $A_{\infty}$-bimodule over $\mathcal{H o m}(D, A)$ by means of (2). Then, the collection of morphisms

$$
F_{p, q}: \mathcal{H o m}(D, A)^{\otimes p} \otimes(M \otimes C) \otimes \mathcal{H o m}(D, A)^{\otimes q} \rightarrow M \otimes D
$$

given by

$$
\begin{aligned}
& F_{p, q}\left(\phi_{1} \otimes \cdots \otimes \phi_{p} \otimes(m \otimes c) \otimes \psi_{1} \otimes \cdots \otimes \psi_{q}\right) \\
& \quad=(-1)^{\varepsilon^{\prime}}\left(\phi_{1}\left(d_{(q+2)}\right) \cdots \phi_{p}\left(d_{(q+p+1)}\right)\right) \cdot m \cdot\left(\psi_{1}\left(d_{(1)}\right) \cdots \psi_{q}\left(d_{(q)}\right)\right) \otimes d_{(q+1)},
\end{aligned}
$$

where $f_{p+q+1}(c)=d_{(1)} \otimes \cdots \otimes d_{(p+q+1)}$, and $\varepsilon^{\prime}$ is defined by the same expression as $\epsilon^{\prime}$ in (6) replacing all occurrences of $c_{(i)}$ by $d_{(i)}$, defines a morphism of $A_{\infty}$-bimodules over $\mathcal{H o m}(D, A)$.

\section{Twists of $A_{\infty}$-algebras}

We will now recall the twisting procedure of $A_{\infty}$-algebras by a Maurer-Cartan element, which was introduced in [6, Chs. 3 and 4] (see also [5], and [10, Ch. 6]). 


\subsection{Topological definitions}

The following definitions are standard. A topology on a $\mathrm{dg}$ vector space $M$ is a decreasing sequence $\left\{F^{i} M\right\}_{i \in \mathbb{N}_{0}}$ of dg vector subspaces of $M$, where $F^{0} M=M$ (cf. [4, Ch. III, $\left.\left.\S 2, \mathrm{n}^{\circ} 5\right]\right)$. We understand each $\mathrm{dg}$ vector subspace $F^{i} M$ as a neighborhood of the zero element of $M$, and we say that $M$ is a topological $d g$ vector space. Note that $F^{i} M$ is closed for all $i \in \mathbb{N}_{0}$. The topology is Hausdorff if $\cap_{i \in \mathbb{N}_{0}} F^{i} M=0$. We regard $k$ with the discrete topology, i.e. the one given by the filtration $F^{i} k=\{0\}$ for $i \in \mathbb{N}$. We define a homogeneous morphism of topological dg vector spaces $f: M \rightarrow N$ to be a homogeneous morphism that is continuous. It is said to be contracting if $f\left(F^{i} M\right) \subseteq F^{i} N$, for all $i \in \mathbb{N}_{0}$. Given two topological dg vector spaces $M$ and $N$, the tensor product $M \otimes N$ has the topology

$$
F^{i}(M \otimes N)=\sum_{i_{1}+i_{2} \geqslant i} F^{i_{1}} M \otimes F^{i_{2}} N
$$

for $i \in \mathbb{N}_{0}$. We remark that this topology is equivalent to the one considered in $[\mathbf{1 4}$, $1.5]$, since

$$
F^{i}(M \otimes N) \subseteq F^{\lfloor i / 2\rfloor} M \otimes N+M \otimes F^{\lfloor i / 2\rfloor} N
$$

and

$$
F^{i} M \otimes N+M \otimes F^{j} N \subseteq F^{\min (i, j)}(M \otimes N),
$$

where $\lfloor x\rfloor \in \mathbb{Z}$ denotes the floor function of $x \in \mathbb{R}$. The definition of completion of a topological dg vector space $M$ is the standard one via inverse limits

$$
\hat{M}=\lim _{\leftarrow \mathbb{N}_{0}} M / F^{j} M, \text { where } F^{i} \hat{M}=\lim _{\leftarrow \mathbb{N}_{0}} F^{i} M / F^{j} M
$$

gives the topology ( $\left.c f .\left[4, \mathrm{Ch} . \mathrm{III}, \S 2, \mathrm{n}^{\circ} 6\right]\right)$. Moreover, the completed tensor product $M \hat{\otimes} N$ is the completion of $M \otimes N$ for the previous topology (8).

We remark that there is an obvious notion of complete $d g$ coalgebra, which is just a coalgebra in the monoidal category of complete topological dg vector spaces and continuous morphisms of dg vector spaces (of degree zero) provided with completed tensor products (see [10, Subsection 6.2.1]). The same comments apply to their (complete) dg (bi)comodules.

All the previous definitions can also be made for the category of graded vector spaces, and they are obtained from the previous ones by imposing the differentials to be zero.

\subsection{Topological $A_{\infty}$-algebras and their bimodules}

A topological augmented $A_{\infty}$-algebra is an augmented $A_{\infty}$-algebra such that the underlying graded vector space has a Hausdorff topology satisfying that all morphisms $m_{i}$, for $i \in \mathbb{N}$, and $\epsilon_{A}$ are contracting morphisms of topological graded vector spaces. We note that the unit morphism $\eta_{A}$ is always contracting. A morphism of topological augmented $A_{\infty}$-algebras $f_{\bullet}: A \rightarrow B$ is a morphism of augmented $A_{\infty}$-algebras such that $f_{i}$ is a contracting morphism of topological graded vector spaces for all $i \in \mathbb{N}$.

The next statement is easy to prove.

Proposition 3.1. Let $V$ be a topological graded vector space with $F^{1} V=V$, and let $C(V)$ be the complete coaugmented graded coalgebra $\prod_{n \in \mathbb{N}_{0}} V^{\hat{\otimes} n}$ with coproduct 
given by deconcatenation, the obvious counit and coaugmentation, and the filtration $\left\{\prod_{m \in \mathbb{N}_{0}} F^{n}\left(V^{\hat{\otimes} m}\right)\right\}_{n \in \mathbb{N}_{0}}$. Then

(i) the topology stated before coincides with the usual product topology, and, as a consequence, if $\iota: T^{c}(V) \rightarrow C(V)$ denotes the canonical inclusion of the graded tensor coalgebra $T^{c}(V)$, its image is dense in $C(V)$;

(ii) $\Delta_{C(V)} \circ \iota=(\iota \otimes \iota) \circ \Delta_{T^{c}(V)}$ and $\iota$ commutes with the corresponding coaugmentations and counits.

In particular, if $V$ is complete, $C(V)$ is the cofree counitary (but not necessarily coaugmented) topological coalgebra over $V$ in the monoidal category of complete topological vector spaces provided with continuous morphisms, i.e. given $C$ any complete topological counitary graded coalgebra $C$ and a continuous morphism $f: C \rightarrow V$, there exists a unique morphism of topological counitary graded coalgebras $F: C \rightarrow C(V)$ such that $\pi_{1} \circ F=f$, where $\pi_{1}: C(V) \rightarrow V$ is the canonical projection. It is explicitly given by

$$
F(c)=\epsilon_{C}(c)+f(c)+\sum_{n \in \mathbb{N}_{\geqslant 2}} f^{\hat{\otimes} n} \circ \Delta_{C}^{(n)} .
$$

Proof. Note that $F^{1} V=V$ yields that $F^{n}\left(V^{\hat{\otimes} m}\right)=V^{\hat{\otimes} m}$ if $m \geqslant n$. Indeed, using identities (8) and (9), $F^{n}\left(V^{\hat{\otimes} m}\right)$ is given as a sum, one of whose terms is precisely $\left(F^{1} V\right)^{\hat{\otimes} n} \hat{\otimes}\left(F^{0} V\right)^{\hat{\otimes}(m-n)}=V^{\hat{\otimes} m}$. As a consequence,

$$
\prod_{m \in \mathbb{N}_{0}} F^{n}\left(V^{\hat{\otimes} m}\right)=\prod_{m=0}^{n-1} F^{n}\left(V^{\hat{\otimes} m}\right) \times \prod_{m \geqslant n} V^{\hat{\otimes} m} .
$$

This is clearly an open neighborhood of the origin of $C(V)$ for the usual product topology, and conversely, any open neighborhood of the origin of $C(V)$ for the usual product topology includes a subspace of the form (10) for $n$ large enough. This implies the first part of item (i). The second part of it follows from [14, Prop. 1.6 and Eq. (1.9)]. Item (ii) is clear. For the last part, see [14, Thm. 3.1].

The following statement is the topological analogue of a well-known result ( $c f$. for instance [11, Lemma 9.1]), and can be regarded as an extension of [6, Lemma 3.2.17].

Lemma 3.2. Let $A$ be a complete Hausdorff topological graded vector space provided with contracting maps $\epsilon_{A}: A \rightarrow k$ and $\eta_{A}: k \rightarrow A$ of degree zero satisfying that $\epsilon_{A} \circ \eta_{A}=\operatorname{id}_{k}$. Let $I_{A}=\operatorname{Ker}\left(\epsilon_{A}\right)$ and $\mathscr{B}(A)=C\left(I_{A}[1]\right)$. Then the set of structures of topological augmented $A_{\infty}$-algebra structures on $A$ with unit $\eta_{A}$ and augmentation $\epsilon_{A}$ is in bijection with the set of contracting coderivations of $\mathscr{B}(A)$ that vanish on the image of the coaugmentation of $\mathscr{B}(A)$ and turn $\mathscr{B}(A)$ into a complete coaugmented dg coalgebra.

Proof. Let $A$ be a topological augmented $A_{\infty}$-algebra, and consider the topology of $I_{A}$ induced by that of $A$, i.e. $F^{p} I_{A}=F^{p} A \cap I_{A}$. By Proposition 3.1, the inclusion $\iota: B^{+}(A) \rightarrow \mathscr{B}(A)$ has dense image. Since the maps $m_{\bullet}$ are contracting, the same is true for $\pi_{1} \circ B$, where $\pi_{1}: B^{+}(A) \rightarrow I_{A}[1]$ is the canonical projection. Hence $\pi_{1} \circ B$ is continuous for the induced topology of $\mathscr{B}(A)$, so it can be thus uniquely extended to a continuous linear map $\hat{b}$ from $\mathscr{B}(A)$ to $I_{A}[1]$. As the filtration defining the topology of $I_{A}[1]^{\otimes n}$ is the completion of that of $I_{A}[1]^{\otimes n}$, and $\pi_{1} \circ B$ is contracting, 
the restriction of $\hat{b}$ to $I_{A}[1]^{\hat{\otimes} n}$ is also contracting. Define $\hat{B}$ from $\mathscr{B}(A)$ to itself as the unique continuous coderivation satisfying that $\left(\hat{\pi}_{1} \circ \hat{B}\right)$ is $\hat{b}$, where $\hat{\pi}_{1}: \hat{B}^{+}(A) \rightarrow$ $I_{A}[1]$ is the canonical projection. Note that $\hat{B} \circ \eta_{\mathscr{B}(A)}=0$ and $\iota: B^{+}(A) \rightarrow \mathscr{B}(A)$ commutes with the differentials. The last property follows from $\hat{\pi}_{1} \circ \hat{B} \circ \iota=\pi_{1} \circ B$ and Proposition 3.1. Hence, $\hat{B} \circ \hat{B} \circ \iota=\iota \circ B \circ B=0$. The continuity of $\hat{B} \circ \hat{B}$ and the density of $\iota$ imply thus that $\hat{B}$ is a differential. This tells us that $\mathscr{B}(A)$ is a complete coaugmented dg coalgebra whose differential vanishes on the image of its coaugmentation and is contracting.

Conversely, if $\hat{B}$ is a contracting coderivation of $\mathscr{B}(A)$ that is a differential and it vanishes on the image of $\eta_{\mathscr{B}(A)}$, we define $b=\hat{\pi}_{1} \circ \hat{B} \circ \iota$, where $\iota: B^{+}(A) \rightarrow \mathscr{B}(A)$ is the canonical inclusion, and extend $b$ to a coderivation $B$ of $B^{+}(A)$. Since $\iota$ commutes with the coaugmentations, $B \circ \eta_{B^{+}(A)}$ vanishes. The fact that $\hat{B}$ is a differential implies that $B$ is also, for $\iota$ commutes with the differentials and it is injective. Moreover, since $\hat{B}$ is contracting, the multiplications $m_{\bullet}$ determined by $B$ are also contracting, and the statement follows.

We shall denote the coaugmented dg coalgebra of the lemma by $\hat{B}^{+}(A)$, and call it the complete bar construction of $A$ (cf. [6, Def. 3.2.16]).

The proof of the next result is similar to the one given for the previous lemma ( $c f$. [11, Lemma 9.3]).

Lemma 3.3. Let $A$ and $A^{\prime}$ be two complete topological augmented $A_{\infty}$-algebras, and let $B^{+}(A)$ and $B^{+}\left(A^{\prime}\right)$ be the corresponding complete bar constructions. Then the set of morphisms $\left\{f_{n}: A^{\otimes n} \rightarrow A^{\prime}\right\}_{n \in \mathbb{N}}$ of topological augmented $A_{\infty}$-algebras is in bijection with the set of contracting morphisms of complete coaugmented dg coalgebras from $\hat{B}^{+}(A)$ to $\hat{B}^{+}\left(A^{\prime}\right)$.

We shall denote the last morphism of the lemma by $\hat{B}^{+}\left(f_{\bullet}\right)$.

Let $M$ be an $A_{\infty}$-bimodule over a complete topological augmented $A_{\infty}$-algebra $A$. We say that $M$ is almost discrete if there exists an increasing filtration $\left\{F_{i} M\right\}_{i \in \mathbb{N}_{0}}$ of $A_{\infty}$-subbimodules of $M$ (i.e. the inclusion $F_{i} M$ in $M$ is a strict morphism of $A_{\infty^{-}}$ bimodules over $A$ ) that is exhaustive (i.e. $\cup_{i \in \mathbb{N}_{0}} F_{i} M=M$ ) and such that, for all $i \in \mathbb{N}_{0}$ there exists $\ell \in \mathbb{N}$ satisfying that $m_{p, q}\left(\omega \otimes m \otimes \omega^{\prime}\right)$ vanishes for all $m \in F_{i} M$, $\omega \in I_{A}[1]^{\otimes p}$ and $\omega^{\prime} \in I_{A}[1]^{\otimes q}$ such that $\omega \otimes \omega^{\prime} \in F^{\ell}\left(I_{A}[1]^{\otimes(p+q)}\right)$. The next result shows that this property is essentially some kind of discreteness assumption on $M$.

Lemma 3.4. Let $\left(A, m_{\bullet}\right)$ be a complete topological augmented $A_{\infty}$-algebra, and let $M$ be a graded vector space provided with an exhaustive increasing filtration $\left\{F_{i} M\right\}_{i \in \mathbb{N}_{0}}$.

If $M$ is an $A_{\infty}$-bimodule over $A$ with structure maps $m_{\bullet}$.. such that each $F_{i} M$ is a strict $A_{\infty}$-subbimodule of $M$ and it is almost discrete with respect to the given filtration, then the bicoderivation $B_{M}$ of the bar construction $B^{+}(A, M, A)$ can be extended to a unique continuous bicoderivation $\hat{B}_{M}$ of the counitary graded bicomodule over $\hat{B}^{+}(A)$ given by $\hat{B}^{+}(A, M, A)=\cup_{i \in \mathbb{N}_{0}} \hat{B}^{+}(A) \hat{\otimes} F_{i} M[1] \hat{\otimes} \hat{B}^{+}(A)$ that respects the filtration and is a differential, where we assume in the latter space that each $F_{i} M$ has the discrete topology and the union has the direct limit topology.

Conversely, assume that there exists a continuous bicoderivation $B^{\prime}$ of the counitary graded bicomodule $\hat{B}^{+}(A, M, A)=\cup_{i \in \mathbb{N}_{0}} \hat{B}^{+}(A) \hat{\otimes} F_{i} M[1] \hat{\otimes} \hat{B}^{+}(A)$ over $\hat{B}^{+}(A)$ that preserves the filtration and is a differential, where each $F_{i} M$ has the discrete 
topology and the union has the direct limit topology. Then $M$ is an almost discrete $A_{\infty}$-bimodule over $A$ with respect to the given filtration and $B^{\prime}$ coincides with the bicoderivation $\hat{B}_{M}$ mentioned previously.

Proof. Suppose that $M$ is almost discrete and set $\pi_{1}^{M}: B^{+}(A, M, A) \rightarrow M[1]$ the projection given by $\epsilon_{B^{+}(A)} \otimes \operatorname{id}_{M[1]} \otimes \epsilon_{B^{+}(A)}$. Let $\iota_{M}: B^{+}(A, M, A) \rightarrow \hat{B}^{+}(A, M, A)$ denote the canonical inclusion. We will show that $\pi_{1}^{M} \circ B_{M}$ can be extended to a unique continuous linear map $\hat{b}_{M}$ from $\hat{B}^{+}(A, M, A)$ to $M[1]$ as follows. Since $F_{i} M$ is a strict $A_{\infty}$-subbimodule of $M, \pi_{1}^{M} \circ B_{M}$ sends $B^{+}(A) \otimes F_{i} M[1] \otimes B^{+}(A)$ to $F_{i} M$. The assumption that $M$ is almost discrete tells us that the previous map vanishes on $F^{\ell}\left(B^{+}(A) \otimes F_{i} M[1] \otimes B^{+}(A)\right)$ for some $\ell$, so it is continuous for the topology induced from $\hat{B}^{+}(A) \hat{\otimes} F_{i} M[1] \hat{\otimes} \hat{B}^{+}(A)$. Since a continuous morphism of graded vector spaces is uniformly continuous, it can be uniquely extended to the completion, and we obtain thus a continuous morphism $\hat{b}_{M}^{i}$ from $\hat{B}^{+}(A) \hat{\otimes} F_{i} M[1] \hat{\otimes} \hat{B}^{+}(A)$ to $F_{i} M[1]$. As a continuous bicoderivation of the latter space over the complete dg coalgebra $\hat{B}^{+}(A)$ is determined by the continuous morphism from $\hat{B}^{+}(A) \hat{\otimes} F_{i} M[1] \hat{\otimes} \hat{B}^{+}(A)$ to $F_{i} M[1]$ given by composition with the canonical projection ( $c f$. [10, Lemme 2.1.2.1]), $\hat{b}_{M}^{i}$ determines a unique continuous bicoderivation $\hat{B}_{M}^{i}$ on $\hat{B}^{+}(A) \hat{\otimes} F_{i} M[1] \hat{\otimes} \hat{B}^{+}(A)$. Finally, $\hat{B}_{M}^{i} \circ \hat{B}_{M}^{i}$ vanishes. Indeed, this follows from the verification of a well-known condition on the map $\hat{\pi}_{1}^{M} \circ \hat{B}_{M}^{i}$ that is analogous to the one in [10, Lemme 2.1.2.1], where $\hat{\pi}_{1}^{M}$ denotes $\epsilon_{\hat{B}^{+}(A)} \hat{\otimes} \mathrm{id}_{M[1]} \hat{\otimes} \epsilon_{\hat{B}^{+}(A)}$. The mentioned identity for $\hat{b}_{M}^{i}$ is just the continuous extension of the analogous condition for the map $\pi_{1}^{M} \circ B_{M}$ restricted to $F_{i} M$, which holds for $B_{M}$ is a differential. Now, it is trivial to see that all the maps $\hat{b}_{M}^{i}$ (or $\hat{B}_{M}^{i}$ ) are compatible for all $i \in \mathbb{N}_{0}$, so they define a unique continuous bicoderivation of $\hat{B}^{+}(A, M, A)$ over $\hat{B}^{+}(A)$ which is also a differential. It is clear that the inclusion $\iota_{M}$ of $B^{+}(A, M, A)$ into $\hat{B}^{+}(A, M, A)$ commutes with the differentials.

Conversely, if there is a continuous bicoderivation $B^{\prime}$ of the counitary graded bicomodule $\hat{B}^{+}(A, M, A)=\cup_{i \in \mathbb{N}_{0}} \hat{B}^{+}(A) \hat{\otimes} F_{i} M[1] \hat{\otimes} \hat{B}^{+}(A)$ over $\hat{B}^{+}(A)$ that respects the filtration and is a differential, where each $F_{i} M$ has the discrete topology and the union hast the direct limit topology, $\hat{\pi}_{1}^{M} \circ B^{\prime}$ induces for each $i \in \mathbb{N}_{0}$ a continuous map from $\hat{B}^{+}(A) \hat{\otimes} F_{i} M[1] \hat{\otimes} \hat{B}^{+}(A)$ to $F_{i} M[1]$. The fact that $F_{i} M$ is discrete implies that there exists $\ell \in \mathbb{N}$ such that $\hat{\pi}_{1}^{M} \circ B^{\prime}$ vanishes on $F^{\ell}\left(\hat{B}^{+}(A) \hat{\otimes} F_{i} M[1] \hat{\otimes} \hat{B}^{+}(A)\right)$. Define $b^{i}$ as the composition of the inclusion of $B^{+}(A) \otimes F_{i} M[1] \otimes B^{+}(A)$ inside $\hat{B}^{+}(A) \hat{\otimes} F_{i} M[1] \hat{\otimes} \hat{B}^{+}(A)$ together with $\hat{\pi}_{1}^{M}$, and take $B^{i}$ as the unique bicoderivation of $B^{+}(A) \otimes F_{i} M[1] \otimes B^{+}(A)$ over $B^{+}(A)$ induced by $b^{i}$. Then $F_{i} M$ is an $A_{\infty^{-}}$ bimodule for all $i \in \mathbb{N}_{0}$ such that the inclusion $F_{i} M \rightarrow F_{i+1} M$ is a strict morphism of $A_{\infty}$-bimodules. This induces the structure of an $A_{\infty}$-bimodule on their union $M$, which is almost discrete with respect to the filtration $\left\{F_{i} M\right\}_{i \in \mathbb{N}_{0}}$. The rest of the proof follows from the comments in the previous paragraph.

Let $M$ and $N$ be two almost discrete $A_{\infty}$-bimodules over a complete topological augmented $A_{\infty}$-algebra $A$ with respect to the increasing filtrations $\left\{F_{i} M\right\}_{i \in \mathbb{N}_{0}}$ and $\left\{F_{i} N\right\}_{i \in \mathbb{N}_{0}}$, respectively, and let $f_{\bullet, \bullet}: M \rightarrow N$ be a morphism of $A_{\infty}$-bimodules over $A$. We say that $f_{\bullet, \bullet}$ is almost discrete (with respect to the given filtrations) if for each $i \in \mathbb{N}_{0}, f_{p, q}\left(I_{A}[1]^{p} \otimes F_{i} M \otimes I_{A}[1]^{q}\right) \subseteq F_{i} N$ for all $p, q \in \mathbb{N}_{0}$, and there is $\ell \in \mathbb{N}$ such that $f_{p, q}\left(\omega \otimes m \otimes \omega^{\prime}\right)$ vanishes for all $m \in F_{i} M, \omega \in I_{A}[1]^{\otimes p}$ and $\omega^{\prime} \in I_{A}[1]^{\otimes q}$ such that $\omega \otimes \omega^{\prime} \in F^{\ell}\left(I_{A}[1]^{\otimes(p+q)}\right)$. 
The proof of the following result is analogous to the one of the previous lemma.

Lemma 3.5. Let $\left(A, m_{\bullet}\right)$ be a complete topological augmented $A_{\infty}$-algebra, and let $\left(M, m_{\bullet \bullet \bullet}^{M}\right)$ and $\left(N, m_{\bullet}^{N}\right)$ be two almost discrete $A_{\infty}$-bimodules over it, with fixed increasing filtrations.

If $f_{\bullet, \bullet}:\left(M, m_{\bullet}^{M}\right) \rightarrow\left(N, m_{\bullet, \bullet}^{N}\right)$ is almost discrete, then the morphism of counitary $d g$ bicomodules $B^{+}\left(f_{\bullet}, \bullet\right)$ can be extended to a unique continuous morphism of counitary $d g$ bicomodules $\hat{B}^{+}\left(f_{\bullet}, \bullet\right)$ from $\hat{B}^{+}(A, M, A)$ to $\hat{B}^{+}(A, N, A)$, that respects the filtration stated in Lemma 3.4 and where we consider the topologies indicated in that result.

Conversely, given a continuous morphism of counitary $d g$ bicomodules $F^{\prime}$ from $\hat{B}^{+}(A, M, A)$ to $\hat{B}^{+}(A, N, A)$ that respects the filtrations mentioned previously, where we consider the topologies indicated there, it induces a unique almost discrete morphism $f_{\bullet, \bullet}:\left(M, m_{\bullet}^{M}, \bullet\right) \rightarrow\left(N, m_{\bullet, \bullet}^{N}\right)$ of $A_{\infty}$-bimodules such that $F^{\prime}$ coincides with the morphism $\hat{B}^{+}\left(f_{\bullet, \bullet}\right)$.

\subsection{Twists of topological $A_{\infty}$-algebras and $A_{\infty}$-bimodules}

Let $A$ be a topological augmented $A_{\infty}$-algebra, which we assume to be $A$ complete.

We recall that $k$ is a complete counitary dg coalgebra with the discrete topology and zero differential. Fix an element $a$ in the neighborhood $F^{1} A$ of cohomological degree 1 and zero Adams degree, and define a morphism of topological graded counitary (not necessarily coaugmented) coalgebras $\xi_{a}: k \rightarrow \hat{B}^{+}(A)$ as the unique one satisfying that $\hat{\pi}_{1} \circ \xi_{a}$ sends $1_{k}$ to $-[a]$, where $\hat{\pi}_{1}: \hat{B}^{+}(A) \rightarrow I_{A}[1]$ is the canonical projection. It exists by the universal property of $\hat{B}^{+}(A)$ stated in Proposition 3.1. We say that $a$ satisfies the (topological) Maurer-Cartan equation if $\xi_{a}$ commutes with the differentials, i.e. $\hat{B} \circ \xi_{a}=0$, and we shall denote the set of such elements by $\operatorname{MC}(A)$. Equivalently, $a$ satisfies the Maurer-Cartan equation if the sum

$$
\sum_{n \in \mathbb{N}}(-1)^{\frac{n(n+1)}{2}} m_{n}\left(a^{\otimes n}\right)
$$

converges to zero. Note that the previous sum always converges because $A$ is complete.

Let $a \in \mathrm{MC}(A)$. Let $t_{a}: \hat{B}^{+}(A) \rightarrow I_{A}[1]$ be the sum of $\hat{\pi}_{1}$ and the composition of the canonical projection $\epsilon_{\hat{B}^{+}(A)}: \hat{B}^{+}(A) \rightarrow k$ together with the map sending $1_{k}$ to $-[a]$. Then, there exists a unique morphism of topological counitary graded coalgebras $T_{a}: \hat{B}^{+}(A) \rightarrow \hat{B}^{+}(A)$ such that $\hat{\pi}_{1} \circ T_{a}=t_{a}$. Note that $T_{a}$ is contracting, $T_{a} \circ \eta_{\hat{B}^{+}(A)}=\xi_{a}$ and that $\hat{\pi}_{1} \circ T_{a} \circ T_{-a}=\hat{\pi}_{1} \circ T_{-a} \circ T_{a}=\hat{\pi}_{1}$. The last chain of identities and the universal property of $\hat{B}^{+}(A)$ imply that $T_{a}$ is an isomorphism with inverse $T_{-a}$.

Given $l, n \in \mathbb{N}_{0}$, define

$$
p_{l, n}^{a}: A^{\otimes n} \rightarrow A^{\otimes(l+n)}
$$

as follows. If $n=0$, we set $p_{l, 0}^{a}\left(1_{k}\right)=a^{\otimes l}$, where $a^{\otimes 0}=1_{k} \in k$. If $n \geqslant 1$,

$$
p_{l, n}^{a}\left(x_{1} \otimes \cdots \otimes x_{n}\right)=\sum_{\bar{l} \in \mathbb{N}_{0}^{n+1, l}}(-1)^{w^{\prime \prime}} a^{\otimes l_{1}} \otimes x_{1} \otimes a^{\otimes l_{2}} \otimes x_{2} \otimes \cdots \otimes a^{\otimes l_{n}} \otimes x_{n} \otimes a^{\otimes l_{n+1}},
$$

where $w^{\prime \prime}=\sum_{j=2}^{n+1} l_{j}\left(\operatorname{deg} x_{1}+\cdots+\operatorname{deg} x_{j-1}+j-1\right)$. Note that $p_{l, n}^{a}$ is a contracting 
morphism of topological graded vector spaces of cohomological degree $l$ and Adams degree zero.

Proposition 3.6. Let $\left(A, m_{\bullet}\right)$ be a complete topological augmented $A_{\infty}$-algebra with strict unit $1_{A}$ and augmentation $\epsilon_{A}$, and let $a \in \mathrm{MC}(A)$. Define

$$
m_{n}^{a}=\sum_{l \in \mathbb{N}_{0}}(-1)^{\frac{l(l+1)}{2}+l n} m_{n+l} \circ p_{l, n}^{a},
$$

for $n \in \mathbb{N}$. It converges by the completeness hypothesis on $A$. Then $\left(A, m_{\bullet}^{a}\right)$ is a topological augmented $A_{\infty}$-algebra with strict unit $1_{A}$ and augmentation $\epsilon_{A}$.

Proof. It is easy to verify that $m_{n}^{a}$ has cohomological degree $2-n$ and zero Adams degree, and it is contracting, for all $n \in \mathbb{N}$. Consider the differentials $B$ and $\hat{B}$ of the bar constructions $B^{+}(A)$ and $\hat{B}^{+}(A)$ of $\left(A, m_{\bullet}\right)$. Define now the new differential $\hat{B}^{a}=T_{a}^{-1} \circ \hat{B} \circ T_{a}$ for $\mathscr{B}(A)$. The fact that $a \in \mathrm{MC}(A)$ implies that $\hat{B}^{a} \circ \eta_{\hat{B}^{+}(A)}=0$, so $\hat{B}^{a}$ is a continuous coderivation of the coaugmented graded coalgebra structure of $\mathscr{B}(A)$. Furthermore, it is contracting, because $\hat{B}, T_{a}$ and its inverse are so. The multiplications of the topological $A_{\infty}$-algebra structure it determines by means of Lemma 3.2 are exactly the ones given in (12). The statement thus follows from Lemma 3.2.

We shall denote the topological augmented $A_{\infty}$-algebra described in the previous proposition by $\left(A, m_{\bullet}^{a}\right)$. It will be called the twisted topological augmented $A_{\infty}$-algebra of $\left(A, m_{\bullet}\right)$ by $a$. Note that these definitions coincide with the corresponding ones for augmented dg algebras, in the case that $m_{i}$ vanishes for $i \geqslant 3$.

Let $a \in \mathrm{MC}(A)$, and let $f_{\bullet}: A \rightarrow B$ be a morphism of topological augmented $A_{\infty^{-}}$ algebras. Define $b$ as the unique element of $B$ satisfying that $\hat{B}^{+}\left(f_{\bullet}\right) \circ \xi_{a}=\xi_{b}$. We call it the image of $a$ under $f_{\bullet}$. Equivalently, $b$ is defined as the convergent sum

$$
\sum_{n \in \mathbb{N}}(-1)^{\frac{n(n+1)}{2}+1} f_{n}\left(a^{\otimes n}\right) .
$$

Proposition 3.7. Let $f_{\bullet}: A \rightarrow B$ be a morphism of complete topological augmented $A_{\infty}$-algebras, and let $a \in \mathrm{MC}(A)$. Then, the image $b$ of a under $f_{\bullet}$ satisfies the Maurer-Cartan equation.

Proof. This follows directly from

$$
\hat{B}^{\prime} \circ \xi_{b}=\hat{B}^{\prime} \circ \hat{B}^{+}\left(f_{\bullet}\right) \circ \xi_{a}=\hat{B}^{+}\left(f_{\bullet}\right) \circ \hat{B} \circ \xi_{a}=0,
$$

where $\hat{B}^{\prime}$ denotes the differential of $B^{+}(B)$ and $\hat{B}$ that of $B^{+}(A)$.

Proposition 3.8. Let $f_{\bullet}: A \rightarrow B$ be a morphism of complete topological augmented $A_{\infty}$-algebras, and let $a \in \mathrm{MC}(A)$. Take $b$ as the image of a under $f_{\bullet}$ and define

$$
f_{n}^{a}=\sum_{l \in \mathbb{N}_{0}}(-1)^{\frac{l(l+1)}{2}+l n} f_{n+l} \circ p_{l, n}^{a},
$$

for $n \in \mathbb{N}$, which converges by the completeness hypothesis on $B$. Then $f_{\bullet}^{a}$ defines a morphism of topological augmented $A_{\infty}$-algebras from $\left(A, m_{\bullet}^{A, a}\right)$ to $\left(B, m_{\bullet}^{B, b}\right)$. 
Proof. The statement follows from the fact that $f_{\bullet}^{a}$ is the morphism of complete topological augmented $A_{\infty}$-algebras satisfying that $\hat{B}^{+}\left(f_{\bullet}^{a}\right)=T_{b}^{-1} \circ \hat{B}^{+}\left(f_{\bullet}\right) \circ T_{a}$.

Given $l^{\prime}, l^{\prime \prime} \in \mathbb{N}_{0}$ and $n^{\prime}, n^{\prime \prime} \in \mathbb{N}_{0}$, let us define

$$
p_{l^{\prime}, n^{\prime}, l^{\prime \prime} n,^{\prime \prime}}^{a}: A^{\otimes n^{\prime}} \otimes M \otimes A^{\otimes n^{\prime \prime}} \rightarrow A^{\otimes\left(l^{\prime}+n^{\prime}\right)} \otimes M \otimes A^{\otimes\left(l^{\prime \prime}+n^{\prime \prime}\right)}
$$

as $p_{l^{\prime}, n^{\prime}}^{a} \otimes \operatorname{id}_{M} \otimes p_{l^{\prime \prime}, n^{\prime \prime}}^{a}$, where the first and last tensor factors morphisms were introduced at the beginning of this subsection.

The proof of the next result is analogous to the one given for Proposition 3.6.

Proposition 3.9. Let $\left(A, m_{\bullet}\right)$ be a complete topological augmented $A_{\infty}$-algebra, a $\in$ $\mathrm{MC}(A)$ and $\left(M, m_{\bullet}, \bullet\right)$ an almost discrete $A_{\infty}$-bimodule over it. Define

$$
m_{n^{\prime}, n^{\prime \prime}}^{a}=\sum_{l^{\prime}, l^{\prime \prime} \in \mathbb{N}_{0}}(-1)^{\frac{\left(l^{\prime}+l^{\prime \prime}\right)\left(l^{\prime}+l^{\prime \prime}+1\right)}{2}+l^{\prime}\left(n^{\prime}+n^{\prime \prime}+1\right)+l^{\prime \prime} n^{\prime \prime}} m_{n^{\prime}+l^{\prime}, n^{\prime \prime}+l^{\prime \prime}} \circ p_{l^{\prime}, n^{\prime}, l^{\prime \prime}, n^{\prime \prime}}^{a}
$$

for $n^{\prime}, n^{\prime \prime} \in \mathbb{N}_{0}$. Then $\left(M, m_{\bullet, \bullet}^{a}\right)$ is an $A_{\infty}$-bimodule over $\left(A, m_{\bullet}^{a}\right)$.

Proof. Note that (13) is well-defined, since the almost discrete assumption of $M$ implies that $\left\{m_{n^{\prime}+l^{\prime}, n^{\prime \prime}+l^{\prime \prime}} \circ p_{l^{\prime}, n^{\prime}, l^{\prime \prime}, n^{\prime \prime}}^{a}\right\}_{l^{\prime}, l^{\prime \prime} \in \mathbb{N}_{0}}$ is locally finite. Define

$$
\hat{B}_{M}^{a}=\left(T_{a}^{-1} \otimes \mathrm{id}_{M[1]} \otimes T_{a}^{-1}\right) \circ \hat{B}_{M} \circ\left(T_{a} \otimes \mathrm{id}_{M[1]} \otimes T_{a}\right) .
$$

The statement follows, since $m_{\bullet, \bullet}^{a}$ are the higher multiplications induced by the unique bicoderivation of $B^{+}(A, M, A)$ over $B^{+}\left(A, m_{\bullet}^{a}\right)$ given in Lemma 3.4 associated to $\hat{B}_{M}^{a}$.

The new $A_{\infty}$-bimodule will be denoted by $\left(M, m_{\bullet}^{a} . \bullet\right)$, and it is called the twisted $A_{\infty}$-bimodule of $\left(M, m_{\bullet}, \bullet\right)$ by $a$. Notice that these definitions coincide with the corresponding ones for bimodules over augmented dg algebras, in the case that $m_{i^{\prime}, i^{\prime \prime}}$ vanishes for $i^{\prime}+i^{\prime \prime} \in \mathbb{N}$.

The proof of the next result is analogous to one given in Proposition 3.9 and it is based on Lemma 3.5.

Proposition 3.10. Let $\left(A, m_{\bullet}\right)$ be a complete topological augmented $A_{\infty}$-algebra, $a \in$ $\operatorname{MC}(A),\left(M, m_{\bullet \bullet \bullet}^{M}\right)$ and $\left(N, m_{\bullet \bullet \bullet}^{N}\right)$ be two almost discrete $A_{\infty}$-bimodules over it, and $f_{\bullet, \bullet}: M \rightarrow N$ be a morphism of $A_{\infty}$-bimodules over $A$. We assume that $f_{\bullet \bullet \bullet}$ is almost discrete. Define

$$
f_{n^{\prime}, n^{\prime \prime}}^{a}=\sum_{l^{\prime}, l^{\prime \prime} \in \mathbb{N}_{0}}(-1)^{\frac{\left(l^{\prime}+l^{\prime \prime}\right)\left(l^{\prime}+l^{\prime \prime}+1\right)}{2}+l^{\prime}\left(n^{\prime}+n^{\prime \prime}+1\right)+l^{\prime \prime} n^{\prime \prime}} f_{n^{\prime}+l^{\prime}, n^{\prime \prime}+l^{\prime \prime}} \circ p_{l^{\prime}, n^{\prime}, l^{\prime \prime}, n^{\prime \prime}}^{a},
$$

for $n^{\prime}, n^{\prime \prime} \in \mathbb{N}_{0}$. Then $\left(f_{\bullet, \bullet}^{a}\right)$ is a morphism of $A_{\infty}$-bimodules from $\left(M, m_{\bullet}^{M, \bullet},{ }^{a}\right)$ to $\left(N, m_{\bullet, \bullet}^{N, a}\right)$ over $\left(A, m_{\bullet}^{a}\right)$.

Proof. The expression (14) is well-defined, since the almost discrete assumption of $f_{\bullet, \bullet}$ implies that the family of maps $\left\{f_{n^{\prime}+l^{\prime}, n^{\prime \prime}+l^{\prime \prime}} \circ p_{l^{\prime}, n^{\prime}, l^{\prime \prime}, n^{\prime \prime}}^{a}\right\}_{l^{\prime}, l^{\prime \prime} \in \mathbb{N}_{0}}$ is locally finite. Define

$$
\hat{B}^{+}\left(f_{\bullet}, \bullet\right)^{a}=\left(T_{b}^{-1} \otimes \operatorname{id}_{N[1]} \otimes T_{b}^{-1}\right) \circ \hat{B}^{+}\left(f_{\bullet \bullet \bullet \bullet}\right) \circ\left(T_{a} \otimes \operatorname{id}_{M[1]} \otimes T_{a}\right) .
$$

The statement holds, since $f_{\bullet}^{a}, \bullet$ is the unique morphism of counitary dg bicomodules over $B^{+}\left(A, m_{\bullet}^{a}\right)$ given in Lemma 3.5 associated to $\hat{B}^{+}\left(f_{\bullet}, \bullet\right)^{a}$. 
The following result will be useful in the sequel.

Lemma 3.11. Let $f_{\bullet}: A \rightarrow B$ be a morphism of topological augmented $A_{\infty}$-algebras and $a \in \mathrm{MC}(A)$. Take $b \in B$ the image of a under $f_{\bullet}$. If $\left(N, m_{\bullet}, \bullet\right)$ is an almost discrete $A_{\infty}$-bimodule over $B$, then the identity of $N$ gives a strict isomorphism of $A_{\infty}$ bimodules over $\left(A, m_{\bullet}^{a}\right)$ between the almost discrete $A_{\infty}$-bimodules $\left(f_{\bullet} N,\left(m_{\bullet \bullet \bullet}^{\prime}\right)^{a}\right)$ and $f_{\bullet}^{a}\left(N, m_{\bullet, \bullet}^{b}\right)$, where $m_{\bullet, \bullet}^{\prime}$ is given by $(2)$.

Proof. Using Propositions 3.9 and 3.10, the statement is a direct consequence of the following commuting diagram

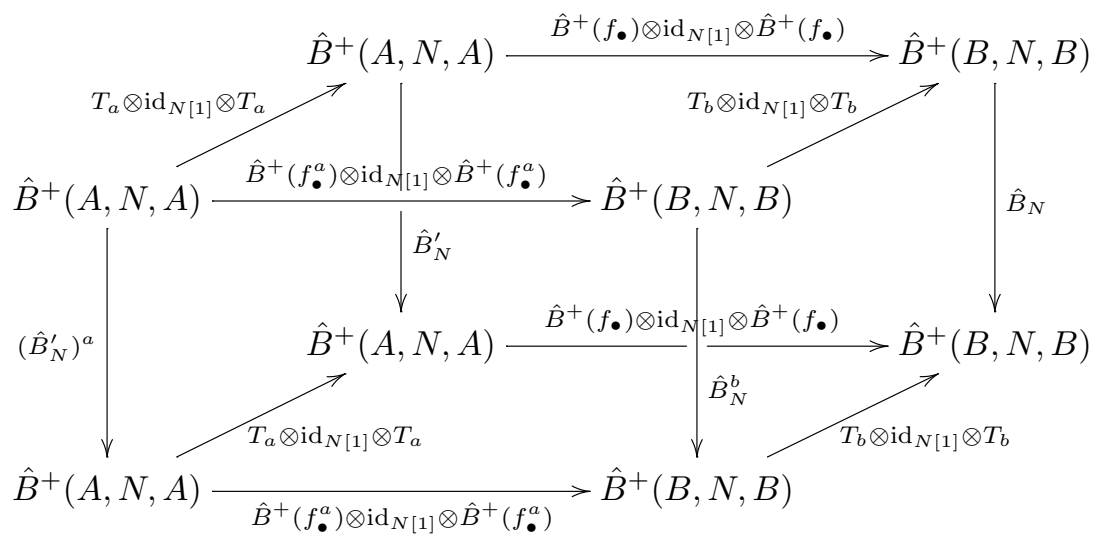

together with Lemmas 3.4 and 3.5. We remark that the commutativity of the back square face is just the completed version of equation (1) (which is a consequence of Lemmas 3.3 and 3.4).

\subsection{The twisted convolution $A_{\infty}$-algebra and the twisted tensor product}

Lemma 3.12. Let $A$ be an augmented $d g$ algebra and $C$ an Adams connected coaugmented $A_{\infty}$-coalgebra. Consider the convolution $A_{\infty}$-algebra $\mathcal{H}=\mathcal{H}$ om $(C, A)$ introduced in Subsection 2.3, and define a topology on it by setting $F^{i} \mathcal{H}$ as the subset of $\mathcal{H}$ given by maps which vanish on the subspace $F_{i-1} C$ of $C$ formed by the sums of the homogeneous elements of Adams degree less than or equal to $i-1$. Then $\mathcal{H}$ is a complete topological augmented $A_{\infty}$-algebra.

Proof. It is easy to see that $\left\{F^{i} \mathcal{H}\right\}_{i \in \mathbb{N}_{0}}$ defines a Hausdorff decreasing filtration of graded vector subspaces of $\mathcal{H}$. We shall now prove that the topology is complete. For all $i \in \mathbb{N}_{0}$, we have the obvious isomorphism of graded vector spaces $\mathcal{H} / F^{i} \mathcal{H} \simeq$ $\mathcal{H o m}\left(F_{i-1} C, A\right)$ induced by $\left.f \mapsto f\right|_{F_{i-1} C}$, for $f \in \mathcal{H}$. This implies that

$$
\lim _{\leftarrow \mathbb{N}_{0}} \mathcal{H} / F^{i} \mathcal{H} \simeq \lim _{\leftarrow \mathbb{N}_{0}} \mathcal{H o m}\left(F_{i-1} C, A\right) \simeq \mathcal{H o m}\left(\lim _{\rightarrow \mathbb{N}_{0}} F_{i-1} C, A\right)=\mathcal{H o m}(C, A) .
$$

Hence, the topological graded vector space $\mathcal{H}$ is complete.

On the other hand, the augmentation of $\mathcal{H}$ is clearly contracting. It thus suffices to prove that the multiplications $m_{n}^{\mathcal{H}}$ are morphisms of topological graded vector spaces for all $n \in \mathbb{N}$. Let $\phi \in F^{i} \mathcal{H}$. Then $m_{1}^{\mathcal{H}}(\phi)=d_{A} \circ \phi-(-1)^{\operatorname{deg} \phi} \phi \circ \Delta_{1}$ vanishes on $F_{i-1} C$, because $\Delta_{1}$ preserves the Adams degree. This implies that $m_{1}^{\mathcal{H}}(\phi) \in F^{i} \mathcal{H}$. 
Let $n \geqslant 2$ and take $\phi_{1} \in F^{i_{1}} \mathcal{H}, \ldots, \phi_{n} \in F^{i_{n}} \mathcal{H}$. By the definition of $m_{n}^{\mathcal{H}}$ given in (3) and the fact that $\Delta_{n}$ preserves the Adams degree, we see that $m_{n}^{\mathcal{H}}\left(\phi_{1}, \ldots, \phi_{n}\right)$ vanishes on any element of $F_{i_{1}+\cdots+i_{n}-1} C$, implying that $m_{n}^{\mathcal{H}}$ is contracting. The lemma is thus proved.

Lemma 3.13. Let $A$ be an augmented dg algebra, $C$ an Adams connected coaugmented $A_{\infty}$-coalgebra, and $M$ a dg bimodule over $A$. Then the $A_{\infty}$-bimodule $M \otimes C$ defined in Subsection 2.3 is almost discrete for the increasing filtration given by $F_{i}(M \otimes C)=M \otimes F_{i} C$, where $F_{i} C$ is the subspace of $C$ formed by the sums of homogeneous elements of Adams degree less than or equal to $i$. Moreover, if $f_{\bullet}: C \rightarrow D$ is a morphism of Adams connected coaugmented $A_{\infty}$-coalgebras, then the induced map described in (7) is almost discrete.

Proof. Let $n^{\prime}, n^{\prime \prime} \in \mathbb{N}_{0}$. Take $m \in M$ and $c \in F_{i} C$ homogeneous, $\omega=\phi_{1} \otimes \cdots \otimes \phi_{p}$ in $F^{a} \mathcal{H o m}(C, A)^{\otimes p}$, and $\omega^{\prime}=\psi_{1} \otimes \cdots \otimes \psi_{q}$ in $F^{b} \mathcal{H o m}(C, A)^{\otimes q}$. Then, using Adams grading considerations and (5) we see that $m_{p, q}\left(\omega \otimes(m \otimes c) \otimes \omega^{\prime}\right)$ vanishes for $a+b \geqslant i+1$, which proves the first part of the lemma. The second part is proved analogously.

A (generalized or homotopical) twisting cochain from $C$ to $A$ is an element $\tau \in$ $\mathcal{H}=\mathcal{H o m}(C, A)$ of cohomological degree 1 and zero Adams degree such that $\epsilon_{A} \circ \tau$ and $\tau \circ \eta_{C}$ vanish and that it satisfies the Maurer-Cartan equation on $\mathcal{H}$. Note that $\tau \circ \eta_{C}=0$ means precisely that $\tau \in F^{1} \mathcal{H}$. Equation (11) translates explicitly into

$$
d_{A} \circ \tau+\sum_{i \in \mathbb{N}}(-1)^{i(i+1) / 2+1} \mu_{A}^{(i)} \circ \tau^{\otimes i} \circ \Delta_{i}=0,
$$

where $\mu_{A}^{(i)}: A^{\otimes i} \rightarrow A$ is the iterative application of the product of $A$. The twist of the convolution $A_{\infty}$-algebra $\mathcal{H o m}(C, A)$ by a twisting cochain $\tau$ will be called the twisted convolution algebra and it will be denoted by $\mathcal{H o m}^{\tau}(C, A)$. The twist of the $A_{\infty}$-bimodule $M \otimes C$ by a twisting cochain $\tau$ will be called the twisted tensor product (of $M$ and $C$ by $\tau$ ) and it will be denoted by $M \otimes_{\tau} C$.

Let $A$ be an augmented dg algebra and $C$ an Adams connected coaugmented $A_{\infty}$ coalgebra. If $\operatorname{Tw}(C, A)$ denotes the set of twisting cochains from $C$ to $A$, we have a canonical map

$$
\operatorname{Hom}_{\text {aug-dg-alg }}\left(\Omega^{+}(C), A\right) \rightarrow \operatorname{Tw}(C, A)
$$

given by $g \mapsto g \circ \tau^{C}$, where $\tau^{C}: C \rightarrow \Omega^{+}(C)$ is the composition of the canonical projection $C \rightarrow C / J_{C}, s_{J_{C}[-1]}^{-1}$ and the canonical inclusion of $J_{C}[-1]$ inside $\Omega^{+}(C)$, where $J_{C}$ is the cokernel of the coaugmentation $\eta_{C}$ of $C$. It is clear that the map (15) is a bijection (see [13, Lemme 3.17]). Furthermore, by means of the previous morphism we can define the composition twisting cochain of a morphism of Adams connected coaugmented $A_{\infty}$-coalgebras $f_{\bullet}: C^{\prime} \rightarrow C$ with a twisting cochain $\tau$ from $C$ to $A$. Indeed, if $F_{\tau} \in \operatorname{Hom}_{\text {aug-dg-alg }}\left(\Omega^{+}(C), A\right)$ is the morphism such that $F_{\tau} \circ \tau^{C}=\tau$, and $\Omega^{+}\left(f_{\bullet}\right)$ is the morphism of augmented dg algebras from $\Omega^{+}\left(C^{\prime}\right)$ to $\Omega^{+}(C)$, the composition twisting cochain $\tau \circ f_{\bullet}$ is defined as $F_{\tau} \circ \Omega^{+}\left(f_{\bullet}\right) \circ \tau^{C^{\prime}}$. It is easy to see that the composition twisting cochain coincides exactly with the image of $\tau$ under the morphism $\left(f_{\bullet}\right)_{*}: \mathcal{H o m}(C, A) \rightarrow \mathcal{H o m}\left(C^{\prime}, A\right)$ introduced in (4). 


\section{Hochschild (co)homology of nonnegatively graded connected algebras}

\subsection{Some preparatory results}

Let $C$ be a coaugmented $A_{\infty}$-coalgebra and $A$ be a nonnegatively (Adams) graded connected algebra, which we regard in zero cohomological degree. Denote by $A^{\mathrm{e}}$ the enveloping algebra of $A$ defined as $A^{\text {op }} \otimes A$. It has two commuting $A$-bimodule actions: the outer structure given by $a \cdot\left(a^{\prime} \otimes b^{\prime}\right) \cdot b=a \cdot a^{\prime} \otimes b^{\prime} . b$, and the inner one of the form $a \cdot\left(a^{\prime} \otimes b^{\prime}\right) \cdot b=a^{\prime} \cdot b \otimes a \cdot b^{\prime}$, for $a, a^{\prime}, b, b^{\prime} \in A$ and where the products of the right members of the two definitions are in $A$.

The next result is clear.

Proposition 4.1. Let $C$ be a coaugmented $A_{\infty}$-coalgebra, let $A$ be a nonnegatively (Adams) graded connected algebra, which we regard in zero cohomological degree, and let $\tau: C \rightarrow A$ be a twisting cochain. Then $A^{\mathrm{e}} \otimes_{\tau} C$ is a complex of free graded $A$ bimodules provided with a map of complexes of $A$-bimodules from it to $A$ of the form $\left(a \otimes a^{\prime}\right) \otimes c \mapsto a^{\prime} . a . \epsilon_{C}(c)$. The previous twisted tensor product is formed by making use of the outer A-bimodule structure of $A^{\mathrm{e}}$, whereas the remaining inner structure turns $A^{\mathrm{e}} \otimes_{\tau} C$ into an A-bimodule. Moreover,

(i) if we apply the functor $\mathcal{H o m}_{A^{\mathrm{e}}}(-, A)$ to $A^{\mathrm{e}} \otimes_{\tau} C$, the complex obtained is naturally identified with the underlying complex of the twisted convolution $A_{\infty}$-algebra $\mathcal{H o m}^{\tau}(C, A)$ given by twisting the topological $A_{\infty}$-algebra of Lemma 3.12;

(ii) if $M$ is a(n Adams) graded A-bimodule, which we regard in zero cohomological degree, the complex obtained by applying functor $M \otimes_{A^{\mathrm{e}}}(-)$ to $A^{\mathrm{e}} \otimes_{\tau} C$ is naturally identified with the underlying complex of the twisted tensor product $A_{\infty}$-bimodule $M \otimes_{\tau} C$ over $\mathcal{H o m}^{\tau}(C, A)$, given by twisting the almost discrete $A_{\infty}$-bimodule of Lemma 3.13.

We recall the following theorem, which must be well-known by the experts. It was announced by B. Keller at the X ICRA of Toronto, Canada, in 2002.

Theorem 4.2. Let $C$ be a minimal coaugmented $A_{\infty}$-coalgebra and $A$ be a nonnegatively (Adams) graded connected algebra, which we regard in zero cohomological degree. Then, the following are equivalent:

(i) There is a quasi-isomorphism of augmented minimal $A_{\infty}$-algebras

$$
\mathcal{E} x t_{A}^{\bullet}(k, k) \rightarrow C^{\#} \text {. }
$$

(ii) There is a twisting cochain $\tau: C \rightarrow A$ such that the twisted tensor product ${ }_{\epsilon_{A}} A \otimes_{\tau} C$ is a minimal projective resolution of the trivial left $A$-module $k$, where the twisted tensor product is formed by using the A-bimodule structure $\epsilon_{A} A$ with the action induced by the augmentation $\epsilon_{A}$ of $A$ on the left and with the standard action on the right, whereas the left $A$-module structure of $\epsilon_{A} A \otimes_{\tau} C$ comes from the remaining left action of $A$.

(iii) There is a twisting cochain $\tau: C \rightarrow A$ such that the twisted tensor product $A^{\mathrm{e}} \otimes_{\tau}$ $C$ is a minimal projective resolution of the regular $A$-bimodule $A$, where the twisted tensor product is formed by using the outer A-bimodule structure of $A^{\mathrm{e}}$, whereas the A-bimodule structure of $A^{\mathrm{e}} \otimes_{\tau} C$ comes from the inner action of the A-bimodule $A^{\mathrm{e}}$. 
Proof. A short proof of the equivalence between (i) and (ii) was given in [8, Thm. 4.7]. Furthermore, the condition (iii) implies (ii) by a standard argument. Indeed, since $A^{e} \otimes_{\tau} C$ is a minimal projective resolution of $A$, the cone of the quasi-isomorphism $A^{e} \otimes_{\tau} C \rightarrow A$ is exact. Since an exact complex of projective left $A$-modules is homotopically trivial, there exists a contracting homotopy $s$ for it, which is $A$-linear. Then $\operatorname{id}_{k} \otimes_{A} s$ is a contracting homotopy of the cone of $k \otimes_{A}\left(A^{e} \otimes_{\tau} C\right) \simeq \epsilon_{A} A \otimes_{\tau} C \rightarrow$ $k \otimes_{A} A \simeq k$. To prove that condition (ii) implies (iii), we only have to show that $A^{e} \otimes_{\tau} C$ is exact in positive homological degrees. This follows from [3, Prop. 4.1].

Note that, according to our conventions, the differential of $\epsilon_{A} A \otimes_{\tau} C$ is the map

$$
\begin{aligned}
m_{0,0}^{\epsilon_{A} A \otimes_{\tau} C}= & \operatorname{id}_{A} \otimes \Delta_{1} \\
& +\sum_{i \in \mathbb{N}}(-1)^{\frac{i(i+1)}{2}}\left(\mu_{A}^{(i+1)} \otimes \operatorname{id}_{C}\right) \circ\left(\operatorname{id}_{A} \otimes \tau^{\otimes i} \otimes \operatorname{id}_{C}\right) \circ\left(\operatorname{id}_{A} \otimes \Delta_{i+1}\right) .
\end{aligned}
$$

\subsection{Main result}

We present now the main result of this article.

Theorem 4.3. Let $A$ be a nonnegatively (Adams) graded connected algebra, that we regard in zero (co)homological degree, let $C$ be a minimal coaugmented $A_{\infty}$-coalgebra such that there is a quasi-isomorphism of augmented minimal $A_{\infty}$-algebras

$$
\mathcal{E} x t_{A}^{\bullet}(k, k) \rightarrow C^{\#},
$$

and let $\tau$ be the associated twisted cochain of Theorem 4.2.

Then, there exists a quasi-isomorphism of $A_{\infty}$-algebras from the cochain complex $C^{\bullet}(A, A)$ computing the Hochschild cohomology of $A$ to $\mathcal{H o m}^{\tau}(C, A)$, which, in particular, induces an isomorphism of graded algebras from $H^{\bullet}(A)$ to $H^{\bullet}\left(\mathcal{H o m}^{\tau}(C, A)\right)$.

Given any graded $A$-bimodule $M$ and using the previous quasi-isomorphism of $A_{\infty}$-algebras, there exists a quasi-isomorphism of $A_{\infty}$-bimodules over $C^{\bullet}(A, A)$ from $M \otimes_{\tau} C$ to the chain complex $C_{\bullet}(A, M)$ computing the Hochschild homology of $A$ with coefficients in $M$, which, in particular, induces an isomorphism of graded bimodules from $H_{\bullet}\left(A \otimes_{\tau} C\right)$ to $H_{\bullet}(A, M)$ over $H H^{\bullet}(A)$.

Proof. By dualizing the quasi-isomorphism of augmented $A_{\infty}$-algebras

$$
B^{+}(A)^{\#} \rightarrow \mathcal{E} x t_{A}^{\bullet}(k, k) \rightarrow C^{\#},
$$

we obtain a quasi-isomorphism of Adams connected coaugmented $A_{\infty}$-coalgebras

$$
f_{\bullet}: C \rightarrow B^{+}(A) \text {. }
$$

Note that $B^{+}(A)$ is also Adams connected, for $A$ is nonnegatively graded and connected (see [12, Lemma 2.2]). By the comments in Subsection 2.3, we obtain a morphism $\left(f_{\bullet}\right)_{*}: \mathcal{H o m}\left(B^{+}(A), A\right) \rightarrow \mathcal{H o m}(C, A)$ of augmented $A_{\infty}$-algebras, which are complete topological $A_{\infty}$-algebras by Lemma 3.12. Since $f_{\bullet}$ respects the Adams degree, $\left(f_{\bullet}\right)_{*}$ is contracting, so a morphism of topological augmented $A_{\infty}$-algebras. By taking a look at the proof of Theorem 4.2 we see that the image under $\left(f_{\bullet}\right)_{*}$ of $\tau_{A}$ is $\tau$, which yields a morphism of topological augmented $A_{\infty}$-algebras $\left(f_{\bullet}\right)_{*}^{\tau_{A}}: \mathcal{H o m}^{\tau_{A}}\left(B^{+}(A), A\right) \rightarrow \mathcal{H o m}^{\tau}(C, A)$. On the other hand, by Lemma 3.13 
$f_{\bullet}$ induces an almost discrete morphism of $A_{\infty}$-bimodules over $\mathcal{H o m}\left(B^{+}(A), A\right)$ of the form

$$
\bar{F}_{\bullet, \bullet}:\left(f_{\bullet}\right)_{*}\left(A^{\mathrm{e}} \otimes C\right) \rightarrow A^{\mathrm{e}} \otimes B^{+}(A),
$$

such that $\bar{F}_{0,0}=\mathrm{id}_{A^{\mathrm{e}}} \otimes f_{1}$. We also have an almost discrete morphism of $A_{\infty}$-bimodules over $\mathcal{H o m}\left(B^{+}(A), A\right)$

$$
F_{\bullet, \bullet}:\left(f_{\bullet}\right)_{*}(M \otimes C) \rightarrow M \otimes B^{+}(A),
$$

such that $F_{0,0}=\operatorname{id}_{M} \otimes f_{1}$. By Proposition 3.10 , the twist of $\bar{F}_{\bullet}, \bullet$ by $\tau_{A}$ yields a morphism of $A_{\infty}$-bimodules over $\mathcal{H o m}^{\tau_{A}}\left(B^{+}(A), A\right)$ of the form

$$
\bar{F}_{\bullet, \bullet}^{\tau_{A}:}\left(f_{\bullet}\right)_{*}^{\tau_{A}}\left(A^{\mathrm{e}} \otimes_{\tau} C\right) \rightarrow A^{\mathrm{e}} \otimes_{\tau_{A}} B^{+}(A),
$$

where we are using Lemma 3.11 to identify $\left(f_{\bullet}\right)_{*}^{\tau_{A}}\left(A^{\mathrm{e}} \otimes_{\tau} C\right)$ and the twist by $\tau_{A}$ of $\left(f_{\bullet}\right)_{*}\left(A^{\mathrm{e}} \otimes C\right)$. It is clearly $A^{e}$-linear for the $A$-bimodule structures coming from the corresponding inner actions of $A^{\mathrm{e}}$. We know that the codomain of this map is the usual Hochschild resolution of the $A$-bimodule $A$ (see [9, Fact 2.1]), whereas the domain is the minimal projective resolution of the $A$-bimodule $A$ by the previous theorem. The map $\bar{F}_{0,0}^{\tau_{A}}$ is a morphism between different free resolutions of $A$-bimodules of $A$, so it is a quasi-isomorphism. This implies that $\bar{F}_{\bullet, \bullet}^{\tau_{A}}$ is also a quasi-isomorphism.

By item (i) of Proposition 4.1, $\mathcal{H o m}_{A^{\mathrm{e}}}\left(\bar{F}_{0,0}^{\tau_{A}}, A\right)$ is the first component of the morphism of augmented $A_{\infty}$-algebras $\left(f_{\bullet}\right)_{*}^{\tau_{A}}: \mathcal{H}^{\prime} m^{\tau_{A}}\left(B^{+}(A), A\right) \rightarrow \mathcal{H o m}^{\tau}(C, A)$, which is thus a quasi-isomorphism. The first part of the theorem thus follows. In particular, there is an isomorphism of graded algebras from $H H^{\bullet}(A)$ to $H^{\bullet}\left(\mathcal{H o m}^{\tau}(C, A)\right)$.

Finally, item (ii) of Proposition 4.1 tells us that $\operatorname{id}_{M} \otimes_{A^{\mathrm{e}}} \bar{F}_{0,0}^{\tau_{A}}$ is the first component of the morphism of $A_{\infty}$-bimodules

$$
F_{\bullet, \bullet}^{\tau_{A}}:\left(f_{\bullet}\right)_{*}^{\tau_{A} A}\left(M \otimes_{\tau} C\right) \rightarrow M \otimes_{\tau_{A}} B^{+}(A)
$$

over $\mathcal{H o m}^{\tau_{A}}\left(B^{+}(A), A\right)$ given by twisting (16) by $\tau_{A}$. Hence, $F_{\bullet}^{\tau_{A}} \bullet$ is a quasi-isomorphism. This induces an isomorphism of graded bimodules over $H H^{\bullet}(A)$ from $H_{\bullet}\left(M \otimes_{\tau} C\right)$ to $H_{\bullet}(A, M)$. The theorem is thus proved.

Addendum 4.4. By (3) and (12), we see that the product of $\mathcal{H}=\mathcal{H o m}^{\tau}(C, A)$ is

$$
\begin{gathered}
m_{2}^{\mathcal{H}, \tau}\left(\phi_{1}, \phi_{2}\right)(c)=\sum_{\substack{l \in \mathbb{N}_{0} \\
\bar{l} \in \mathbb{N}_{0}^{3, l}}}(-1)^{\sigma} \tau\left(c_{(1)}\right) \cdots \tau\left(c_{\left(l_{1}\right)}\right) \phi_{1}\left(c_{\left(l_{1}+1\right)}\right) \tau\left(c_{\left(l_{1}+2\right)}\right) \cdots \\
\cdots \tau\left(c_{\left(L_{2}+1\right)}\right) \phi_{2}\left(c_{\left(L_{2}+2\right)}\right) \tau\left(c_{\left(L_{2}+3\right)}\right) \cdots \tau\left(c_{(l+2)}\right),
\end{gathered}
$$

where $\sigma=l+l_{2}+\operatorname{deg} \phi_{1} \operatorname{deg} \phi_{2}+\left(l_{2}+l_{3}\right) \operatorname{deg} \phi_{1}+l_{3} \operatorname{deg} \phi_{2}, L_{2}=l_{1}+l_{2}, \phi_{1}, \phi_{2} \in$ $\mathcal{H}$ and $c \in C$ are homogeneous, and where the sum in the expression of the comultiplication map $\Delta_{l+2}(c)=c_{(1)} \otimes \cdots \otimes c_{(l+2)}$ is omitted for simplicity.

Analogously, the left action of a homogeneous element $\phi \in \mathcal{H}$ on $m \otimes c \in M \otimes_{\tau} C$, that is also assumed to be homogeneous, is obtained by using (5) and (13) for $n^{\prime}=1$ and $n^{\prime \prime}=0$, and it gives

$$
\begin{gathered}
\sum_{\substack{l^{\prime}, l^{\prime \prime} \in \mathbb{N}_{0} \\
\bar{l}^{\prime} \in \mathbb{N}_{0}^{2, l^{\prime}}}}(-1)^{\sigma^{\prime}} \tau\left(c_{\left(l^{\prime \prime}+2\right)}\right) \cdots \tau\left(c_{\left(L_{1}^{\prime}+1\right)}\right) \phi\left(c_{\left(L_{1}^{\prime}+2\right)}\right) \tau\left(c_{\left(L_{1}^{\prime}+3\right)}\right) \cdots \\
\cdots \tau\left(c_{(l+2)}\right) m \tau\left(c_{(1)}\right) \cdots \tau\left(c_{\left(l^{\prime \prime}\right)}\right) \otimes c_{\left(l^{\prime \prime}+1\right)},
\end{gathered}
$$


where $\sigma^{\prime}=l^{\prime \prime}+l_{2}^{\prime}+\operatorname{deg} \phi\left(l_{1}^{\prime}+1\right)+\operatorname{deg} c\left(\operatorname{deg} \phi+l^{\prime}\right), L_{1}^{\prime}=l^{\prime \prime}+l_{1}, l=l^{\prime}+l^{\prime \prime}$, and where the sum in the expression of the comultiplication map $\Delta_{l+2}(c)=c_{(1)} \otimes \cdots \otimes$ $c_{(l+2)}$ is omitted for simplicity.

\subsection{An application}

We shall now provide an application of the previous theorem, showing that the first part gives a different proof of the main result of [15, Thm. 3.2], that computes the cup product for the Hochschild cohomology of any $s$-Koszul algebra. From our point of view, this computation is just a direct consequence of Theorem 4.3 taking into account that the $A_{\infty}$-coalgebra $C$ is just the dual to the $A_{\infty}$-algebra considered in [7, Thm. 6.5], and the twisting cochain is given by minus the composition of the canonical projection $C \rightarrow V$ and the canonical inclusion $V \rightarrow A$. However, our main result may also be applied to compute the algebraic structure of the Hochschild (co)homology of any nonnegatively graded connected algebra, e.g. if $A$ is multi-Koszul in the sense of [8], by applying Thm. 4.8 of that article (taking into account, however, that the definition of Maurer-Cartan equation there has a different sign convention).

Let $V$ be a finite dimensional vector space over $k$ and $A=T V /(R)$ be an $s$ homogeneous algebra for $s \in \mathbb{N}_{\geqslant 2}$, i.e. $R \subseteq V^{\otimes s}$. We say that $A$ is generalized Koszul (or $s$-Koszul, if we want to emphasize the degree of $R$ ) if the minimal projective resolution $P_{\bullet}$ of the trivial left $A$-module $k$ satisfies that $P_{n}$ is (a graded free module) generated in degree $\xi_{s}(n)$, for all $n \in \mathbb{N}_{0}$, where $\xi_{s}(2 m)=s m$ and $\xi_{s}(2 m+1)=s m+1$, for all $m \in \mathbb{N}_{0}$.

The Koszul property of $A$ implies that $C=\operatorname{Tor}_{\bullet}^{A}(k, k)$ satisfies that

$$
C_{p}=\operatorname{Tor}_{p}^{A}(k, k)=\bigcap_{i=0}^{\xi_{s}(p-2)} V^{\otimes i} \otimes R \otimes V^{\otimes\left(\xi_{s}(p-2)-i\right)},
$$

for $p \geqslant 2$, together with $C_{0}=\operatorname{Tor}_{0}^{A}(k, k)=k$ and $C_{1}=\operatorname{Tor}_{1}^{A}(k, k)=V$ (see [2, Eq. (2.5)]). From now on we shall only consider $s>2$, for the case $s=2$ has already been treated in [9, Prop. 2.2]. We give to $C$ the following $A_{\infty}$-coalgebra structure. There are only two nonvanishing comultiplications, $\Delta_{2}$ and $\Delta_{s}$, which satisfy that

(i) $\left.\left(\mathfrak{p}_{p_{1}} \otimes \mathfrak{p}_{p_{2}}\right) \circ \Delta_{2}\right|_{C_{p}}$ is the canonical inclusion if $p_{1}+p_{2}=p$ and $\xi_{s}(p)=\xi_{s}\left(p_{1}\right)+$ $\xi_{s}\left(p_{2}\right)$ for $p_{1}, p_{2}, p \in \mathbb{N}_{0}$, and zero else;

(ii) $\left.\left(\mathfrak{p}_{p_{1}} \otimes \cdots \otimes \mathfrak{p}_{p_{s}}\right) \circ \Delta_{s}\right|_{C_{p}}$ is the canonical inclusion if $p_{1}+\cdots+p_{s}=p+s-2$ and $\xi_{s}(p)=\xi_{s}\left(p_{1}\right)+\cdots+\xi_{s}\left(p_{s}\right)$ for $p_{1}, \ldots, p_{s}, p \in \mathbb{N}$, and zero otherwise;

where $\mathfrak{p}_{m}: C \rightarrow C_{m}$ denotes the canonical projection. Note that the nonvanishing statement of item (i) implies that either $p_{1}$ or $p_{2}$ is even, whereas in the case of item (ii) it yields that $p_{1}, \ldots, p_{s}$ are odd (and $p$ even). It is now trivial to verify ${ }_{\epsilon_{A}} A \otimes_{\tau} C$ is the minimal projective (Koszul) resolution of ${ }_{A} k$, where $\tau: C \rightarrow A$ is the twisting cochain given by the composition of the canonical projection $C \rightarrow V$ together with minus the canonical inclusion. By Thm. 4.2, the graded dual of $C$ is quasi-isomorphic to $\operatorname{Ext}_{A}^{\bullet}(k, k)$, and it is precisely the one appearing in [7, Thm. 6.5].

If $\bar{n}=\left(n_{1}, \ldots, n_{5}\right) \in \mathbb{N}_{0}^{5}$ is a partition of $n \in \mathbb{N}_{0}$, consider the canonical map $a_{\bar{n}}: V^{\otimes n} \rightarrow V^{\otimes n_{1}} \otimes \cdots \otimes V^{\otimes n_{5}}$. Given $n \in \mathbb{N}_{0}$ and $p_{1}, p_{2} \in \mathbb{N}_{0}$ such that $n \geqslant \xi_{s}\left(p_{1}\right)+$ 
$\xi_{s}\left(p_{2}\right)$, define the set of its decompositions of type $\left(p_{1}, p_{2}\right)$ of an element $\omega \in V^{\otimes n}$ as $\left\{a_{\bar{n}}(\omega): \bar{n} \in \mathbb{N}_{0}^{5}\right.$ is a partition of $n$ such that $n_{2 i}=\xi_{s}\left(p_{i}\right)$ for $i=1$ and 2$\}$.

In what follows we shall denote $a_{\bar{n}}(\omega)$ by $\omega_{1} \omega_{1}^{\prime} \omega_{2} \omega_{2}^{\prime} \omega_{3}$.

The next result is a more general statement than the one appearing in $[15$, Thm. 3.2 , which only covers the case of Hochschild cohomology. Our proof is completely different.

Theorem 4.5. Let $A$ be an s-Koszul algebra, with $s \in \mathbb{N}_{>2}$. By Theorem 4.3, the graded algebras $H^{\bullet}(A)$ and $H^{\bullet}\left(\mathcal{H o m}^{\tau}(C, A)\right)$ are isomorphic, and the product $\phi_{1}$. $\phi_{2}$ of two cocycles $\phi_{1} \in \mathcal{H}^{p_{1}}=\mathcal{H o m}\left(C_{p_{1}}, A\right)$ and $\phi_{2} \in \mathcal{H}^{p_{2}}=\mathcal{H o m}\left(C_{p_{2}}, A\right)$ in the latter algebra is described in general by (17). In this particular case, we obtain:

1. if either $p_{1}$ or $p_{2}$ is even, then $\phi_{1} \cdot \phi_{2} \in \mathcal{H}^{p_{1}+p_{2}}$ is

$$
\left(\phi_{1} \cdot \phi_{2}\right)(c)=\phi_{1}\left(c_{(1)}\right) \cdot \phi_{2}\left(c_{(2)}\right),
$$

where $c=c_{(1)} \otimes c_{(2)}, c_{(1)} \in C_{p_{1}}$ and $c_{(2)} \in C_{p_{2}}$, and we have omitted the sum in the expression of $c$;

2. if both $p_{1}$ and $p_{2}$ are odd, then $\phi_{1} \cdot \phi_{2} \in \mathcal{H}^{p_{1}+p_{2}}$ is

$$
\left(\phi_{1} \cdot \phi_{2}\right)(c)=-(-1)^{s} \sum \omega_{1} \phi_{1}\left(\omega_{1}^{\prime}\right) \omega_{2} \phi_{2}\left(\omega_{2}^{\prime}\right) \omega_{3},^{2}
$$

where the sum is indexed over the set of decompositions $\omega_{1} \omega_{1}^{\prime} \omega_{2} \omega_{2}^{\prime} \omega_{3}$ of type $\left(p_{1}, p_{2}\right)$ of the fixed element $c \in C_{p_{1}+p_{2}}$, for which the sum and tensor symbols are omitted in order to reduce the notation, and $\omega_{i} \in V^{\otimes n_{2 i-1}}$.

Given any graded A-bimodule, Theorem 4.3 tells us that there is an isomorphism of graded bimodules between $H_{\bullet}\left(A \otimes_{\tau} C\right)$ and $H_{\bullet}(A, M)$ over $H H^{\bullet}(A)$. The left action of a cocycle $\phi \in \mathcal{H}^{p}=\mathcal{H o m}\left(C_{p}, A\right)$ on $m \otimes c \in M \otimes C_{q}$ is described in general by (18). In this case, we obtain:

1. if $q$ is odd, or both $p$ and $q$ are even, then $\phi \cdot(m \otimes c) \in M \otimes C_{q-p}$ is

$$
\phi\left(c_{(2)}\right) \cdot m \otimes c_{(1)},
$$

where $c=c_{(1)} \otimes c_{(2)}, c_{(2)} \in C_{p}$, and we have omitted the sum in the expression of $c$;

2. if $p$ is odd and $q$ are even, then $\phi \cdot(m \otimes c) \in M \otimes C_{q-p}$ is

$$
-\sum(-1)^{n_{1}+n_{3}+n_{5}}\left(\omega_{2} \phi\left(\omega_{2}^{\prime}\right) \omega_{3} m \omega_{1}\right) \otimes \omega_{1}^{\prime},
$$

where the sum is indexed over the set of decompositions $\omega_{1} \omega_{1}^{\prime} \omega_{2} \omega_{2}^{\prime} \omega_{3}$ of type $(q-p, p)$ of $c \in C_{q}$ for which the sum and the tensor signs are omitted in order to reduce the notation, and $\omega_{i} \in V^{\otimes n_{2 i-1}}$.

Proof. This is a direct consequence of Theorem 4.3, Addendum 4.4 and the description of the $A_{\infty}$-coalgebra structure of $\operatorname{Tor}_{\bullet}^{A}(k, k)$ given in the previous paragraph.

\footnotetext{
${ }^{2}$ The sign difference with [15] comes from the fact that our definition of cup product respects the Koszul sign rule, whereas the corresponding one in the mentioned article does not.
} 


\section{References}

[1] Luchezar Avramov, Hans-Bjorn Foxby, and Stephen Halperin, Differential graded homological algebra. Preprint.

[2] Roland Berger, Koszulity for nonquadratic algebras, J. Algebra 239 (2001), no. 2, 705-734.

[3] Roland Berger and Nicolas Marconnet, Koszul and Gorenstein properties for homogeneous algebras, Algebr. Represent. Theory 9 (2006), no. 1, 67-97.

[4] N. Bourbaki, Éléments de mathématique. Fascicule XXVIII. Algèbre commutative. Chapitre 3: Graduations, filtrations et topologies. Chapitre 4: Idéaux premiers associés et décomposition primaire, Actualités Scientifiques et Industrielles, No. 1293, Hermann, Paris, 1961 (in French).

[5] Kenji Fukaya, Deformation theory, homological algebra and mirror symmetry, Geometry and physics of branes (Como, 2001), Ser. High Energy Phys. Cosmol. Gravit., IOP, Bristol, 2003, pp. 121-209.

[6] Kenji Fukaya, Yong-Geun Oh, Hiroshi Ohta, and Kaoru Ono, Lagrangian intersection Floer theory: anomaly and obstruction. Part I, AMS/IP Studies in Advanced Mathematics, vol. 46, American Mathematical Society, Providence, RI; International Press, Somerville, MA, 2009.

[7] Ji-Wei He and Di-Ming Lu, Higher Koszul algebras and A-infinity algebras, J. Algebra 293 (2005), no. 2, 335-362.

[8] Estanislao Herscovich, On the multi-Koszul property for connected algebras, Doc. Math. 18 (2013), 1301-1347.

[9] _ Hochschild (co)homology of Koszul dual pairs. Preprint, available at https://www-fourier.ujf-grenoble.fr/ eherscov/Articles/Hochschild(co)homology-of-Koszul-dual-pairs.pdf.

[10] Kenji Lefèvre-Hasegawa, Sur les $A_{\infty}$-catégories, Ph.D. Thesis, Paris, 2003 (in French). Corrections at http://www.math.jussieu.fr/ keller/lefevre/ TheseFinale/corrainf.pdf.

[11] D.M. Lu, J.H. Palmieri, Q.S. Wu, and J.J. Zhang, $A_{\infty}$-algebras for ring theorists, Proceedings of the International Conference on Algebra, 2004, pp. 91-128.

[12] Di Ming Lu, John H. Palmieri, Quan Shui Wu, and James J. Zhang, Koszul equivalences in $A_{\infty}$-algebras, New York J. Math. 14 (2008), 325-378.

[13] Alain Prouté, $A_{\infty}$-structures. Modèles minimaux de Baues-Lemaire et Kadeishvili et homologie des fibrations, Repr. Theory Appl. Categ. 21 (2011), 1-99 (in French). Reprint of the 1986 original; With a preface to the reprint by Jean-Louis Loday.

[14] Mitsuhiro Takeuchi, Topological coalgebras, J. Algebra 97 (1985), no. 2, 505-539.

[15] Yunge $\mathrm{Xu}$ and Huali Xiang, Hochschild cohomology rings of d-Koszul algebras, J. Pure Appl. Algebra 215 (2011), no. 1, 1-12.

Estanislao Herscovich Estanislao.Herscovich@univ-grenoble-alpes.fr

Institut Fourier, Université Grenoble Alpes, 100 rue des Mathématiques, Gières, 38610, France 